\title{
Synthesis of gem-Dimethylcyclopentane-Fused Arenes with Various Topologies via TBD-Mediated Dehydro-Diels-Alder Reaction
}

Liyan Zhang, ${ }^{\text {a,b }}$ Tengda Jin, ${ }^{\mathrm{a}, \mathrm{b}}$ Yingjie Guo, ${ }^{\mathrm{a}, \mathrm{b}}$ Keju Sun, ${ }^{\mathrm{a}, \mathrm{b}}$ A. C. Martin, ${ }^{\mathrm{c}}$ Gregory B. Dudley, ${ }^{* c}$ and Jingyue Yang *a,b

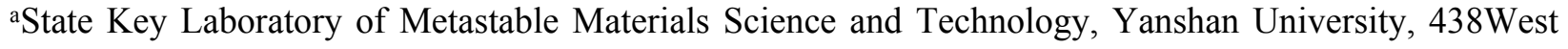
Hebei Street, Qinhuangdao 066004, P. R. China

'Hebei Key Laboratory of Applied Chemistry, School of Environmental and Chemical Engineering, Yanshan University, 438 West Hebei Street, Qinhuangdao 066004, P. R. China

${ }^{\mathrm{c}}$ C. Eugene Bennett Department of Chemistry, West Virginia University, Morgantown, WV, 26506, USA

\section{Supporting Information}

\section{Contents}

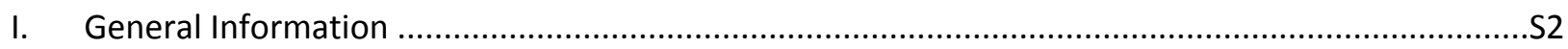

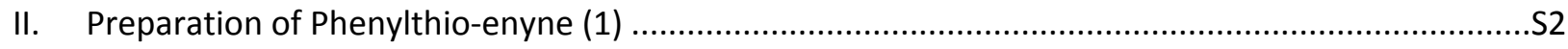

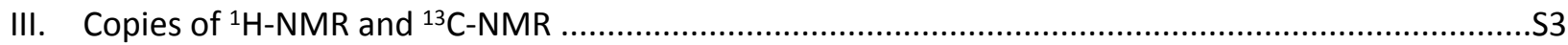

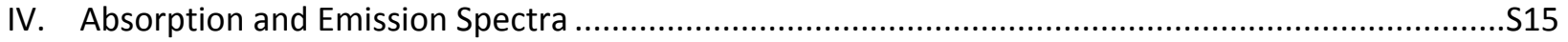

V. Oak Ridge Thermal Ellipsoid Plot diagram of compound 4c ........................................................

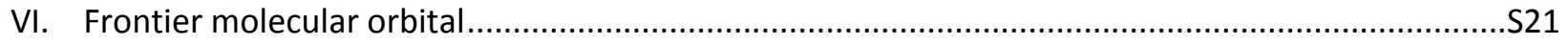

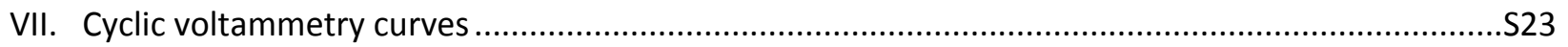

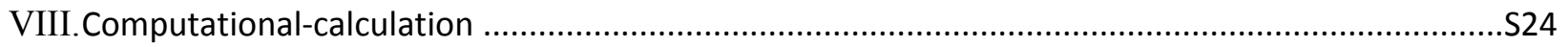

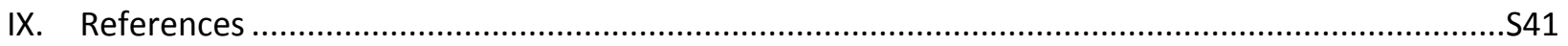




\section{General Information}

${ }^{1} \mathrm{H}$ NMR and ${ }^{13} \mathrm{C}$ NMR spectra were obtained on a $400 \mathrm{MHz}$ Bruker Avance III spectrometer using $\mathrm{CDCl}_{3}$ or $\mathrm{CD}_{2} \mathrm{Cl}_{2}$ as the deuterated solvent. Chemical shifts are reported in parts per million (ppm) relative to TMS (0 ppm for ${ }^{1} \mathrm{H} \mathrm{NMR}$ ), $\mathrm{CDCl}_{3}$ (77.16 ppm for ${ }^{13} \mathrm{C} \mathrm{NMR}$ ) and $\mathrm{CD}_{2} \mathrm{Cl}_{2}$ (5.33 ppm for ${ }^{1} \mathrm{H} \mathrm{NMR}, 53.84 \mathrm{ppm}$ for ${ }^{13} \mathrm{C}$ NMR). Coupling constants $(J)$ are reported in Hertz (Hz). IR spectra were recorded on an FT-IR NICOLET iS10 spectrometer with diamond ATR accessory. High resolution mass data were collected by the Thermo scientific Q Exactive HF (orbitrap). All chemicals were used as received without further purification. Glassware was oven-dried prior to use and all purifications were performed by flash chromatography using silica gel with 200-300 mesh. Absorption spectra data were recorded on an UV-2550 UV-visible spectrophotometer in a quartz cuvette with a path length of $1 \mathrm{~cm}$. Steady-state emission data were collected at room temperature using a Hitachi F-7000 Spectrophotometer with slit widths set at 2.5 nm. Fluorescence quantum yield was obtained on a Hitachi F-7000 Spectrophotometer relative to phenanthrene $\left(0.01 \mathrm{mM}\right.$ in ethanol, $\left.\varphi_{\mathrm{F}}=0.10\right)$. Cyclic voltammetry $(\mathrm{CV})$ was performed on an electrochemical analyzer with a three-electrode cell in a solution of $0.1 \mathrm{M}$ tetrabutylammonium hexafluorophosphate $\left(\mathrm{Bu}_{4} \mathrm{NPF}_{6}\right)$ dissolved in $\mathrm{CH}_{2} \mathrm{Cl}_{2}$. A platinum electrode $\left(0.6 \mathrm{~cm}^{2}\right)$, a platinum electrode $\left(0.5 \mathrm{~cm}^{2}\right)$ and an $\mathrm{Ag} / \mathrm{AgCl}$ electrode were used as the working electrode, counter electrode and reference electrode, respectively. Its potential was calibrated by the ferrocene/ferricenium ( $\mathrm{vs} \mathrm{Ag} / \mathrm{AgCl}$ in $\mathrm{CH}_{2} \mathrm{Cl}_{2}$ ).

\section{Preparation of Phenylthio-enyne (1)}

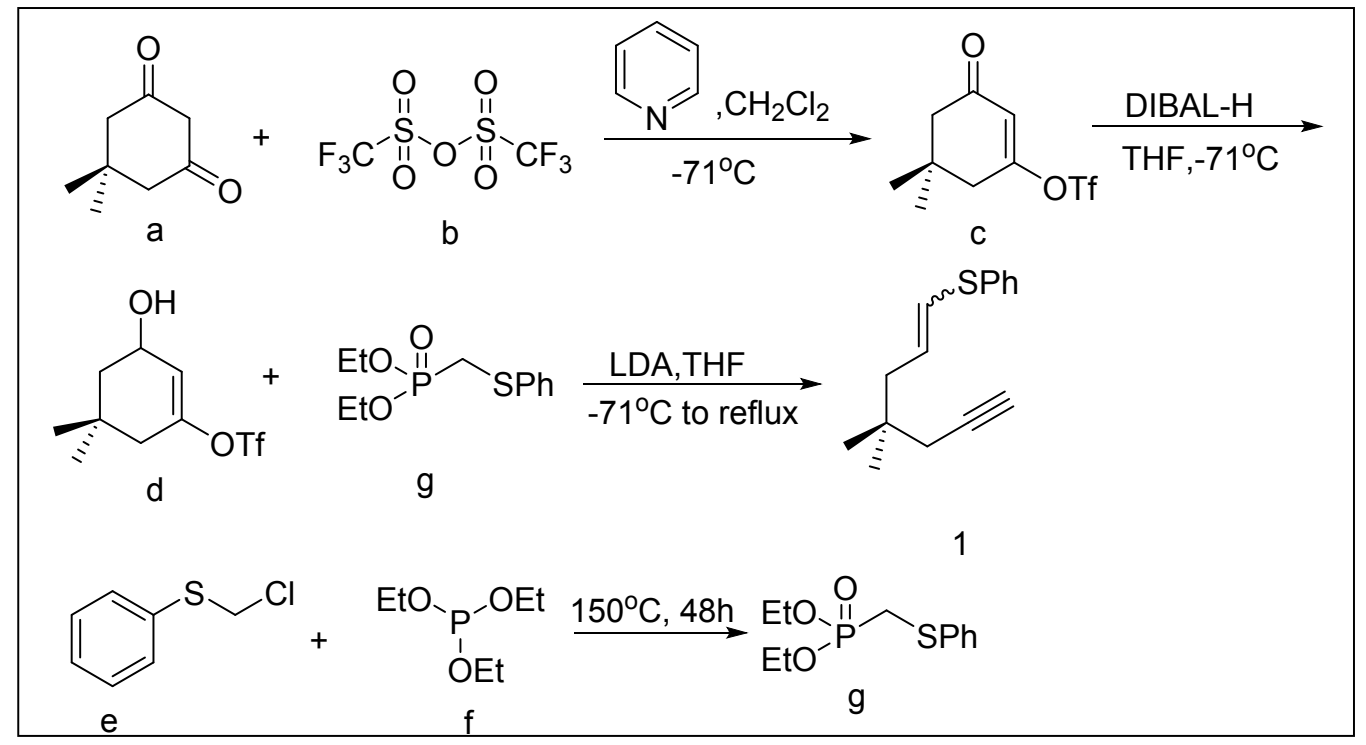

Scheme S1. Synthetic strategy for phenylthio-enyne (1) 


\section{Copies of ${ }^{1} \mathrm{H}-\mathrm{NMR}$ and ${ }^{13} \mathrm{C}-\mathrm{NMR}$}

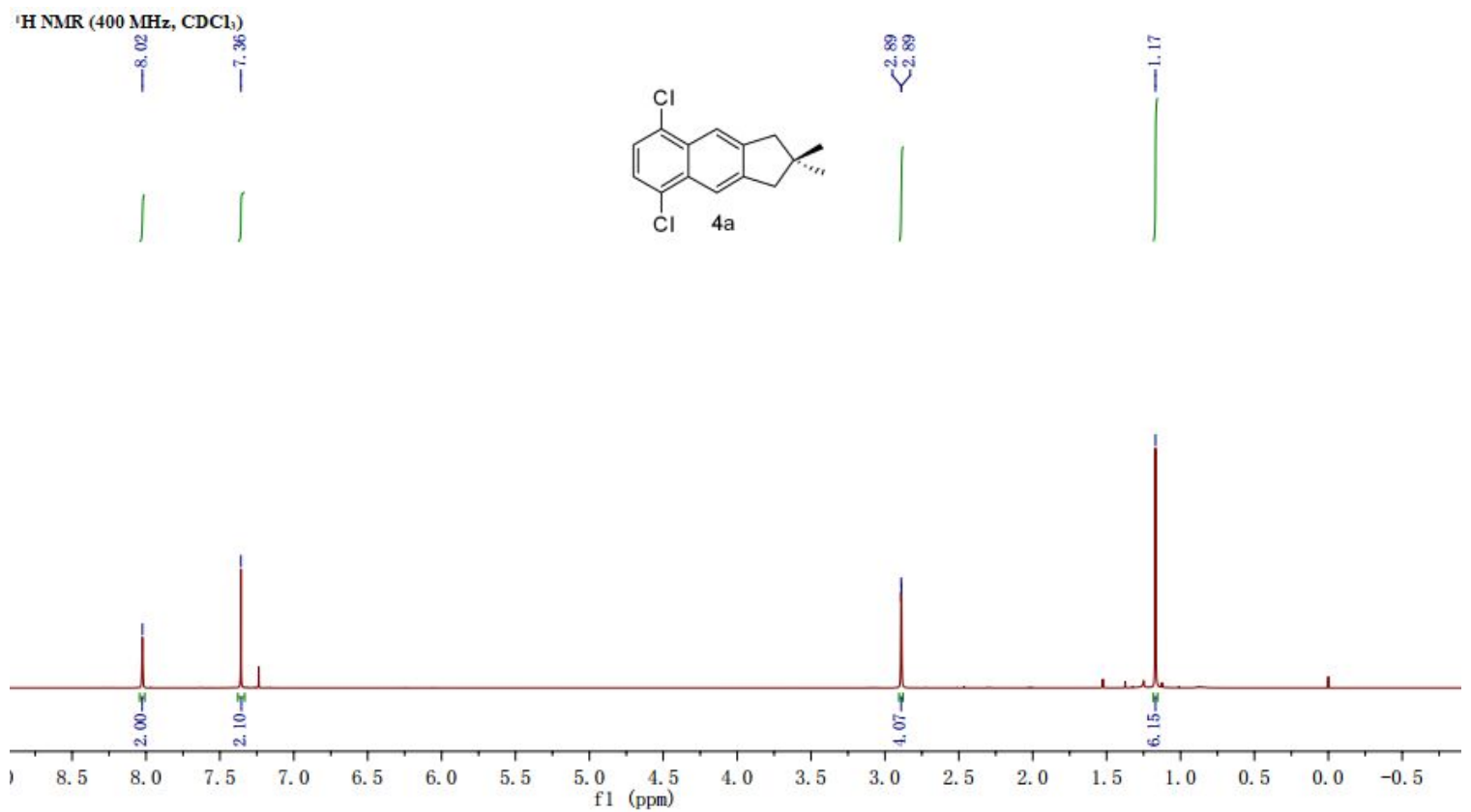

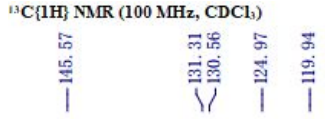
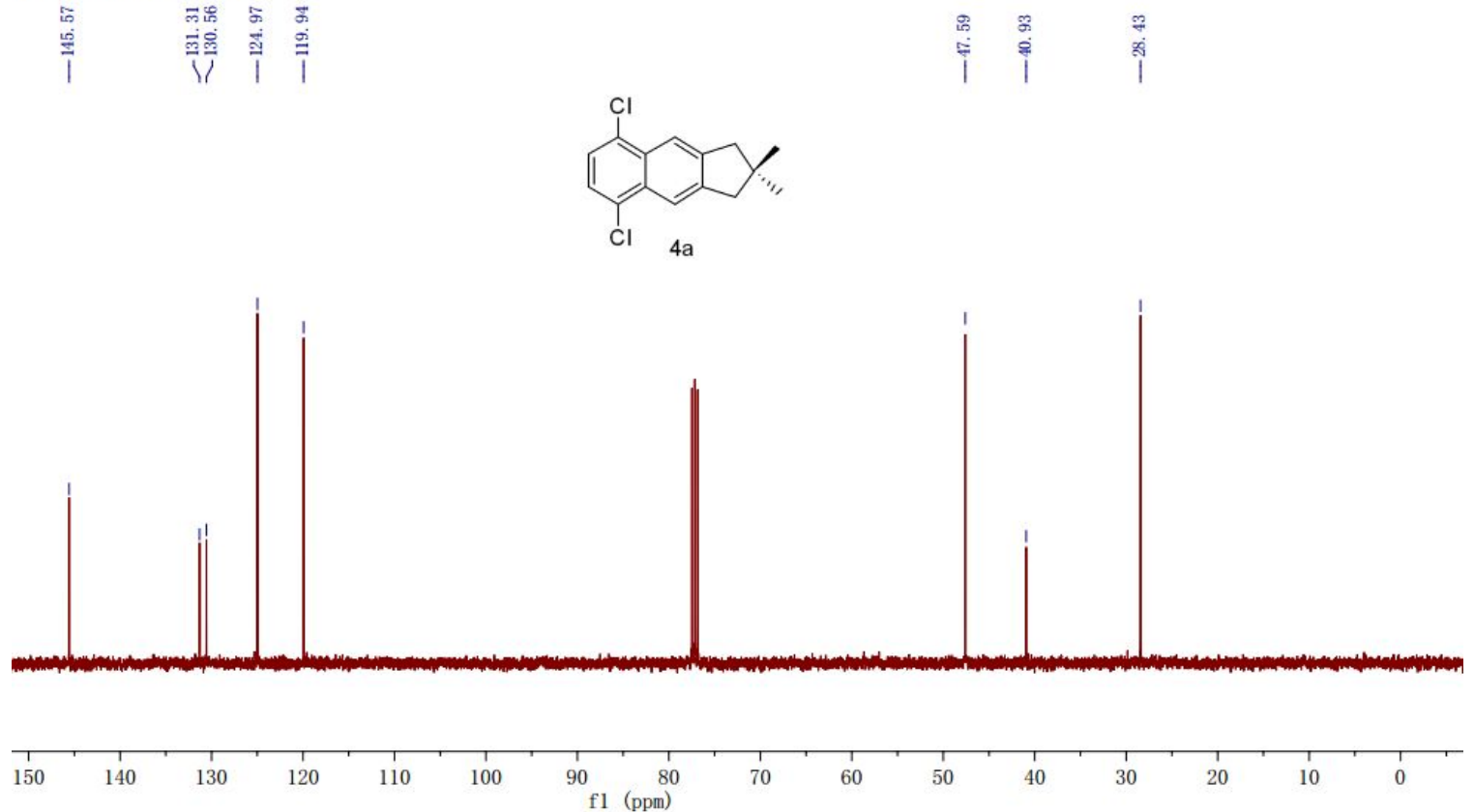
'H NMR (400 MHz, $\mathrm{CDCl}_{3}$ )

ले
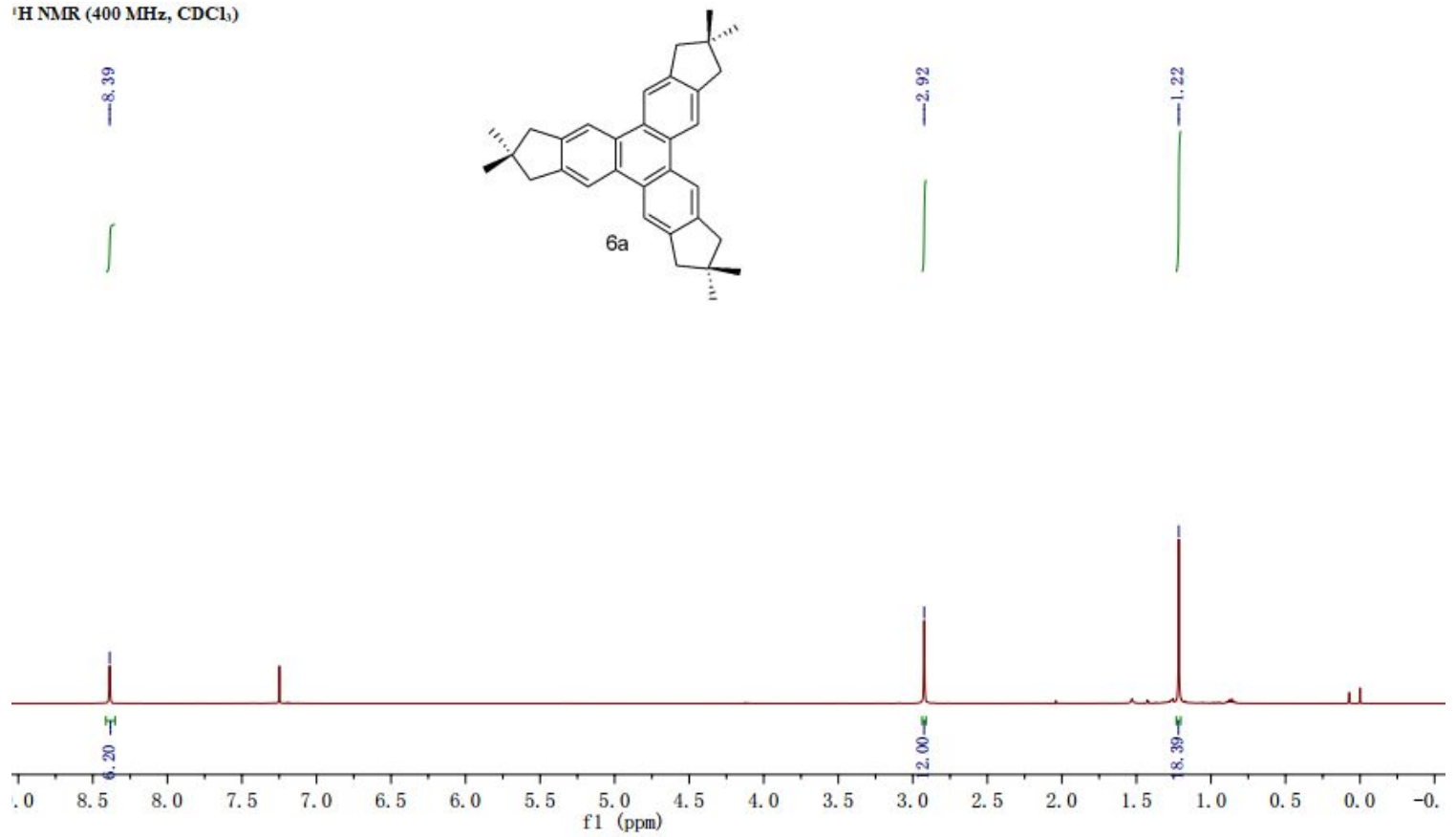

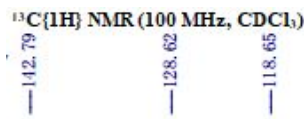
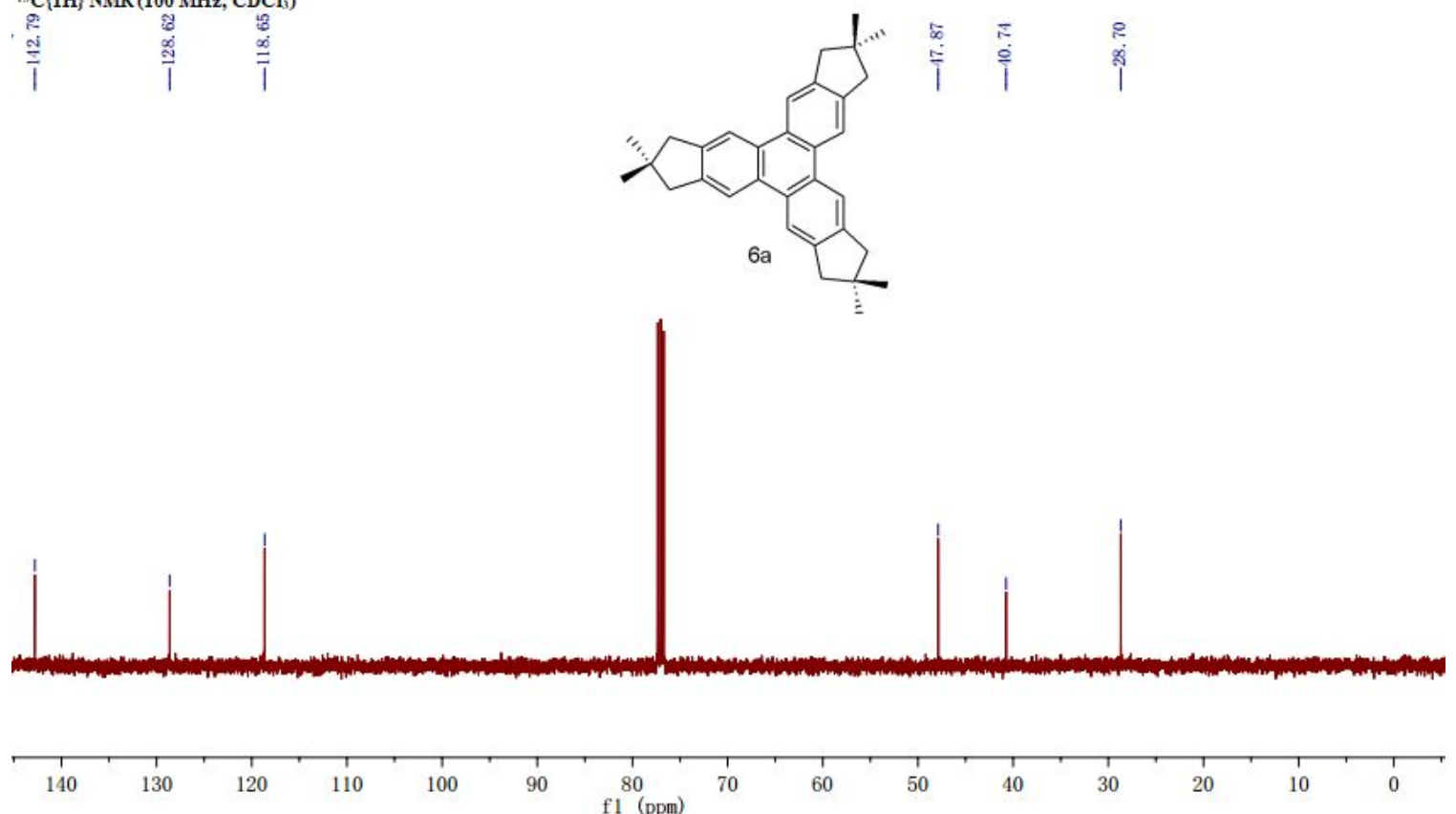
'H NMR (400 MHz, $\left.\mathrm{CDCl}_{3}\right)$

8. 88814 ำ

i vijuting

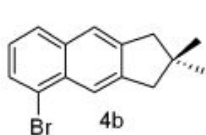

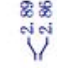

I IIS I

$\mathrm{Br} \quad 4 \mathrm{~b}$

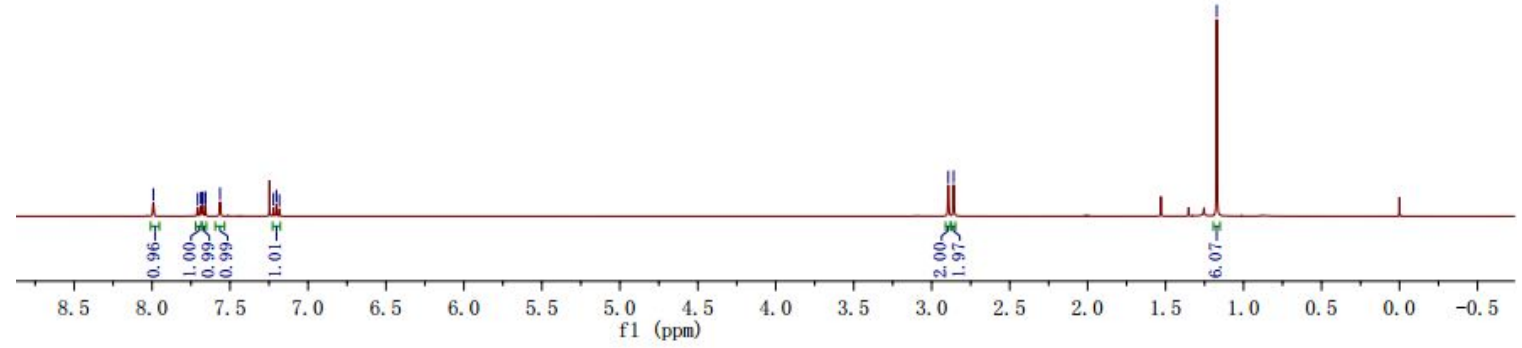

${ }^{13} \mathrm{C}\{\mathrm{lH}\} \operatorname{NMR}\left(100 \mathrm{MHz}, \mathrm{CDCl}_{3}\right)$

용 씨유ำ

主 का
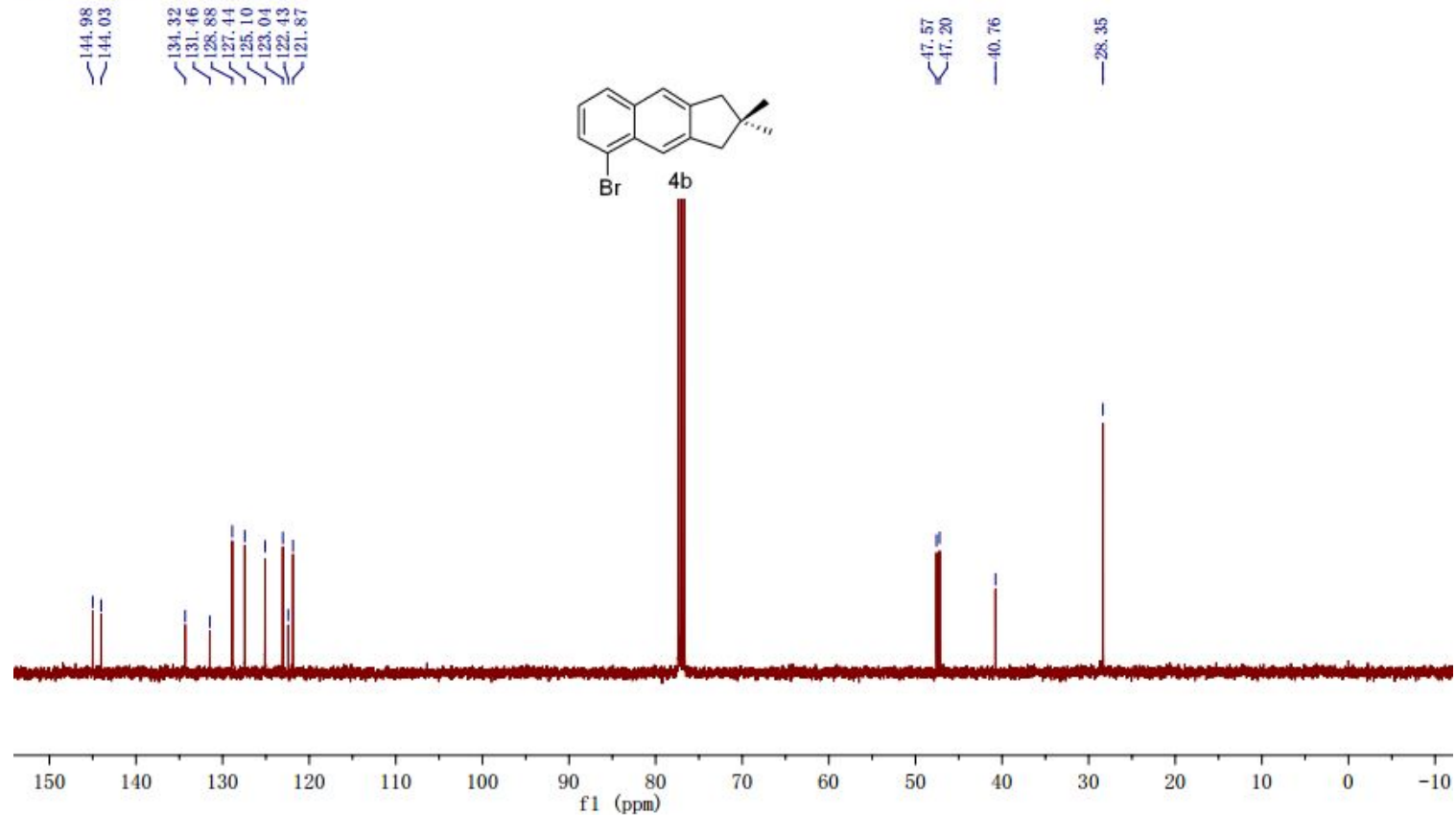


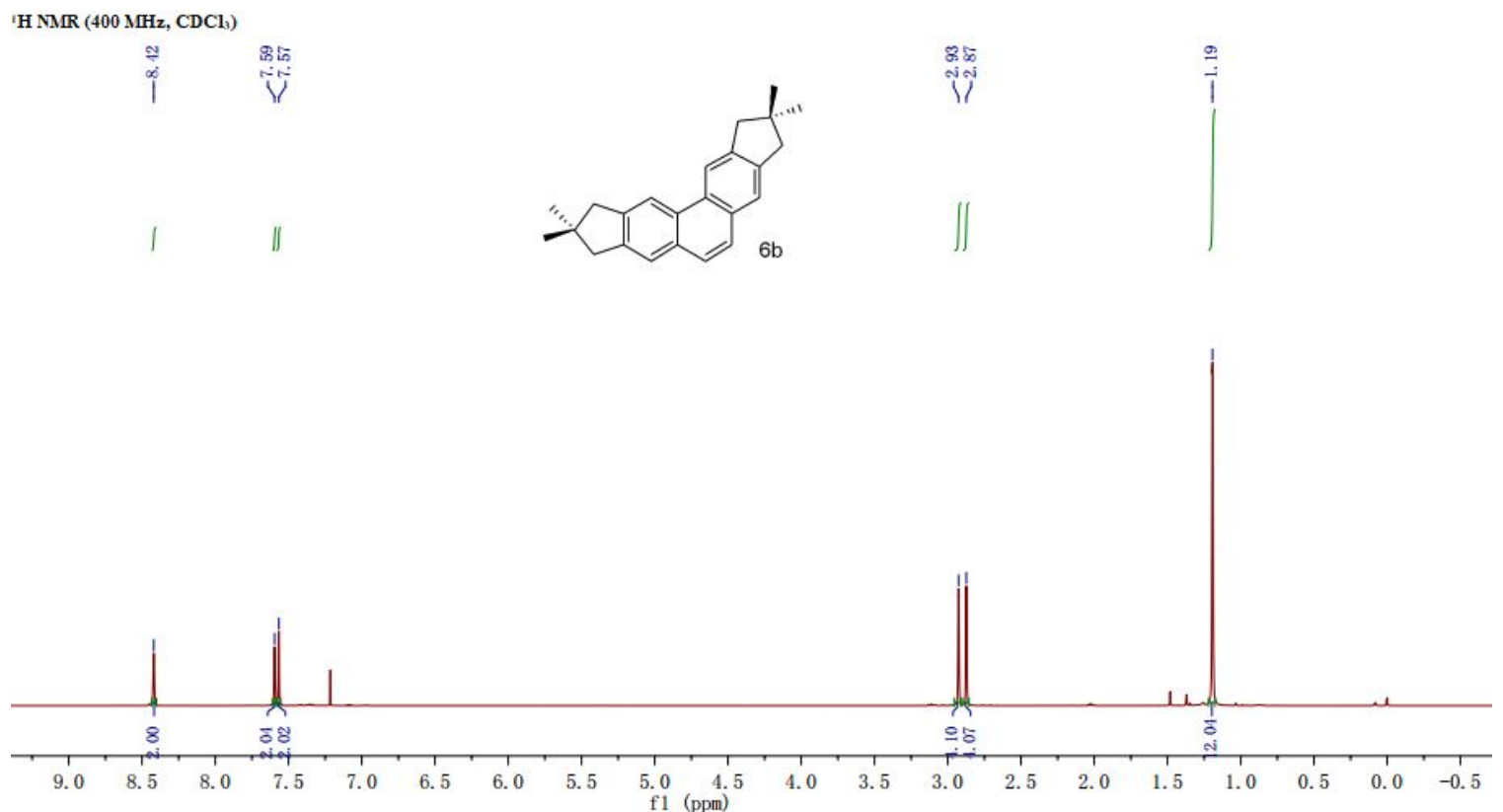

${ }^{13} \mathrm{C}\{1 \mathrm{H}\}$ NMR $\left(100 \mathrm{MHz}, \mathrm{CDCl}_{3}\right)$
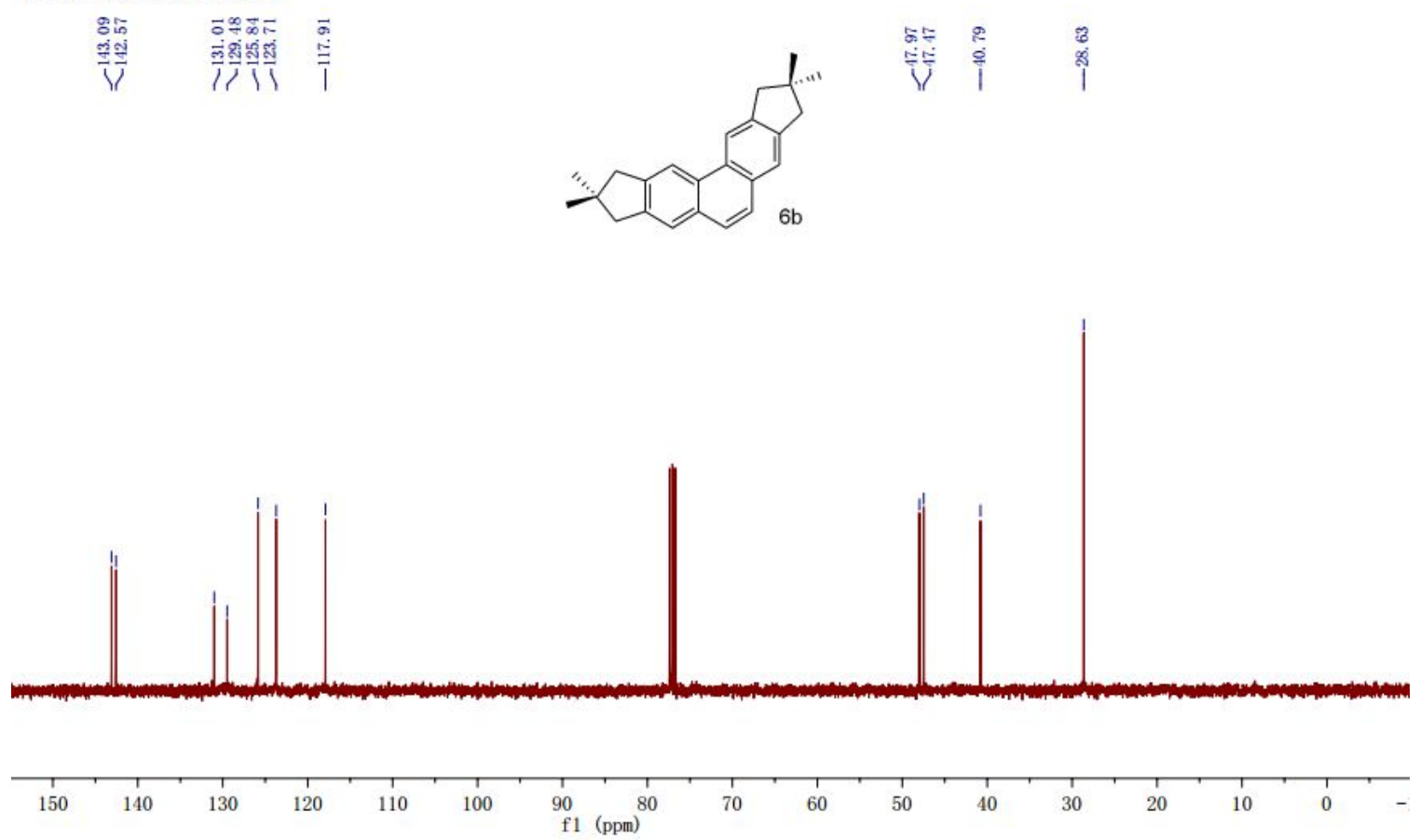
'H NMR (400 MHz, $\left.\mathrm{CD}_{2} \mathrm{Cl}_{2}\right)$

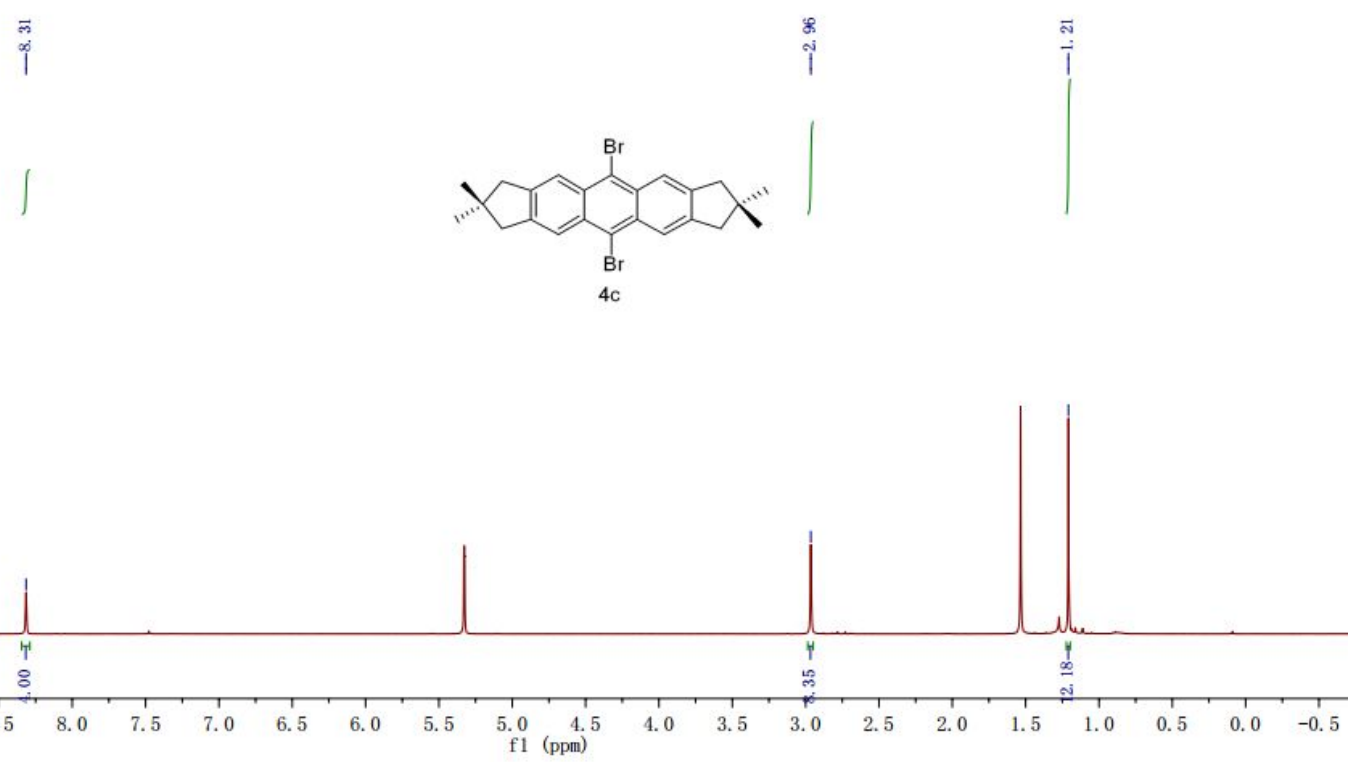

${ }^{13} \mathrm{C}\{1 \mathrm{H}\} \mathrm{NMR}\left(100 \mathrm{MHz}, \mathrm{CD}_{2} \mathrm{Cl}_{2}\right)$

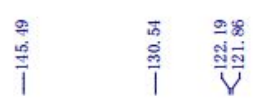
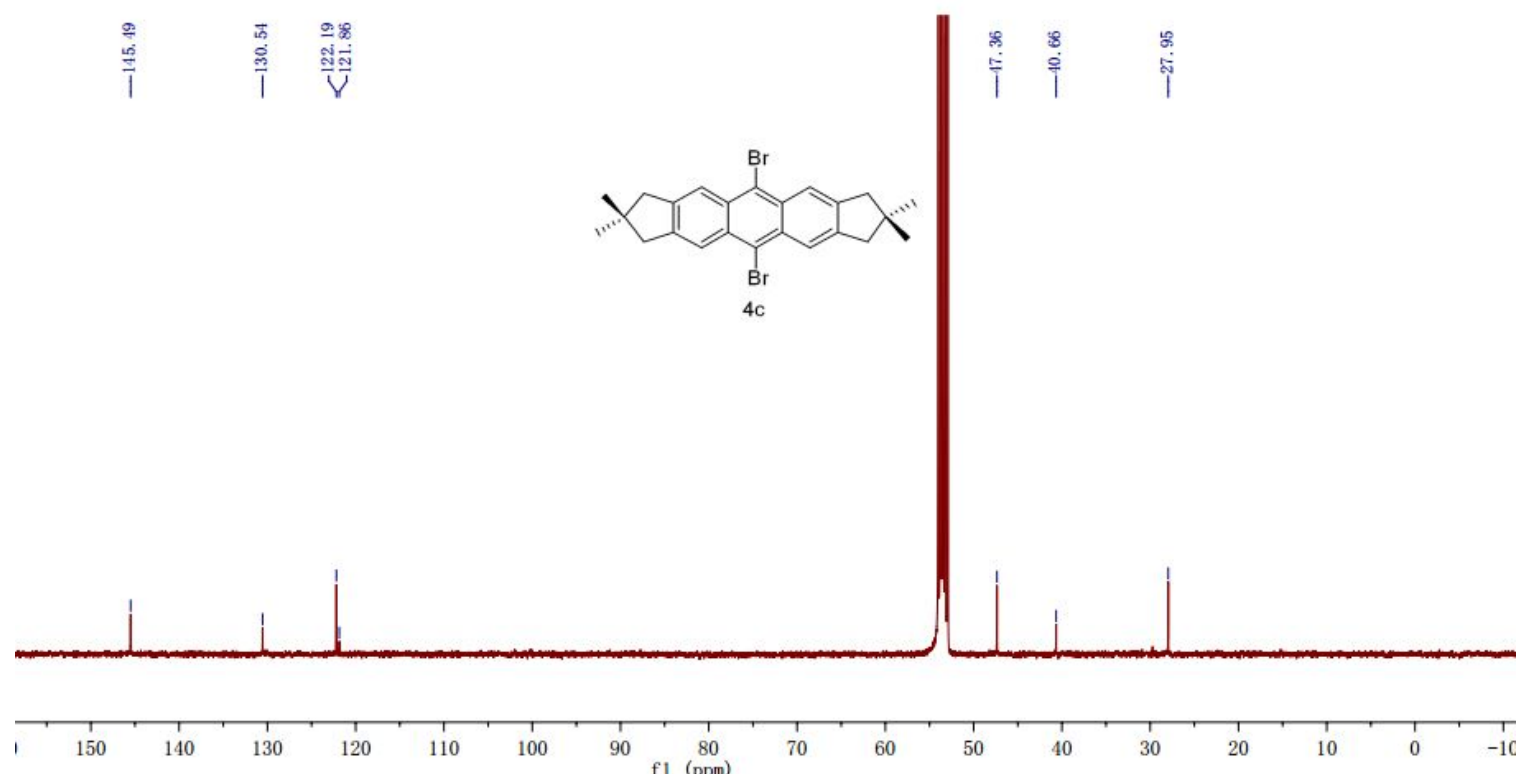
'H NMR (400 MrHz, $\left.\mathrm{CD}_{2} \mathrm{Cl}_{2}\right)$

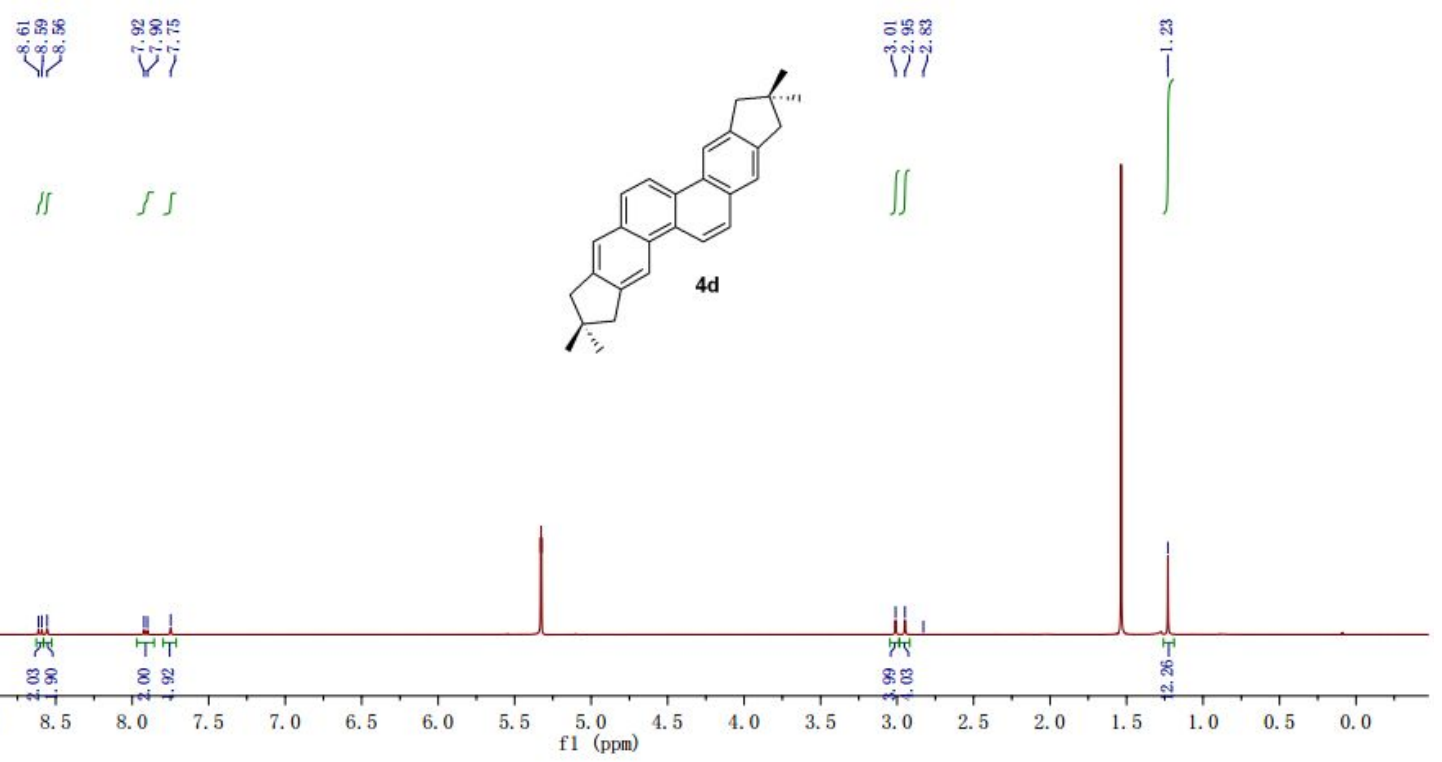


'H NMR (400 MHz, $\mathrm{CDCl}_{3}$ )

$$
\begin{aligned}
& \text { ธำ รูมสล } \\
& \text { ओप } \\
& \text { II II }
\end{aligned}
$$
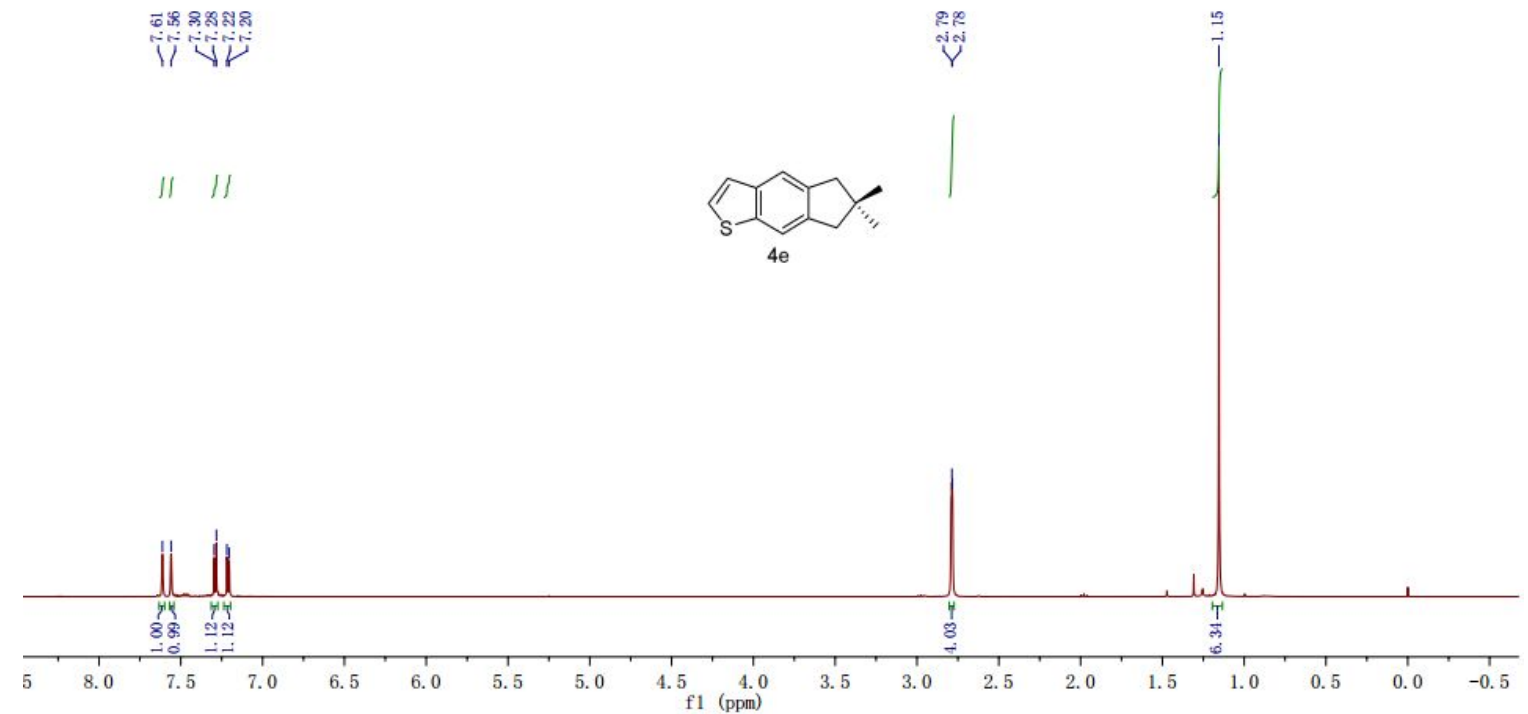

${ }^{13} \mathrm{C}\{1 \mathrm{H}\}$ NMR $\left(100 \mathrm{MHz}, \mathrm{CDCl}_{3}\right)$

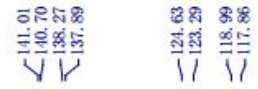

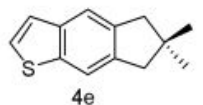

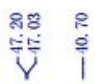
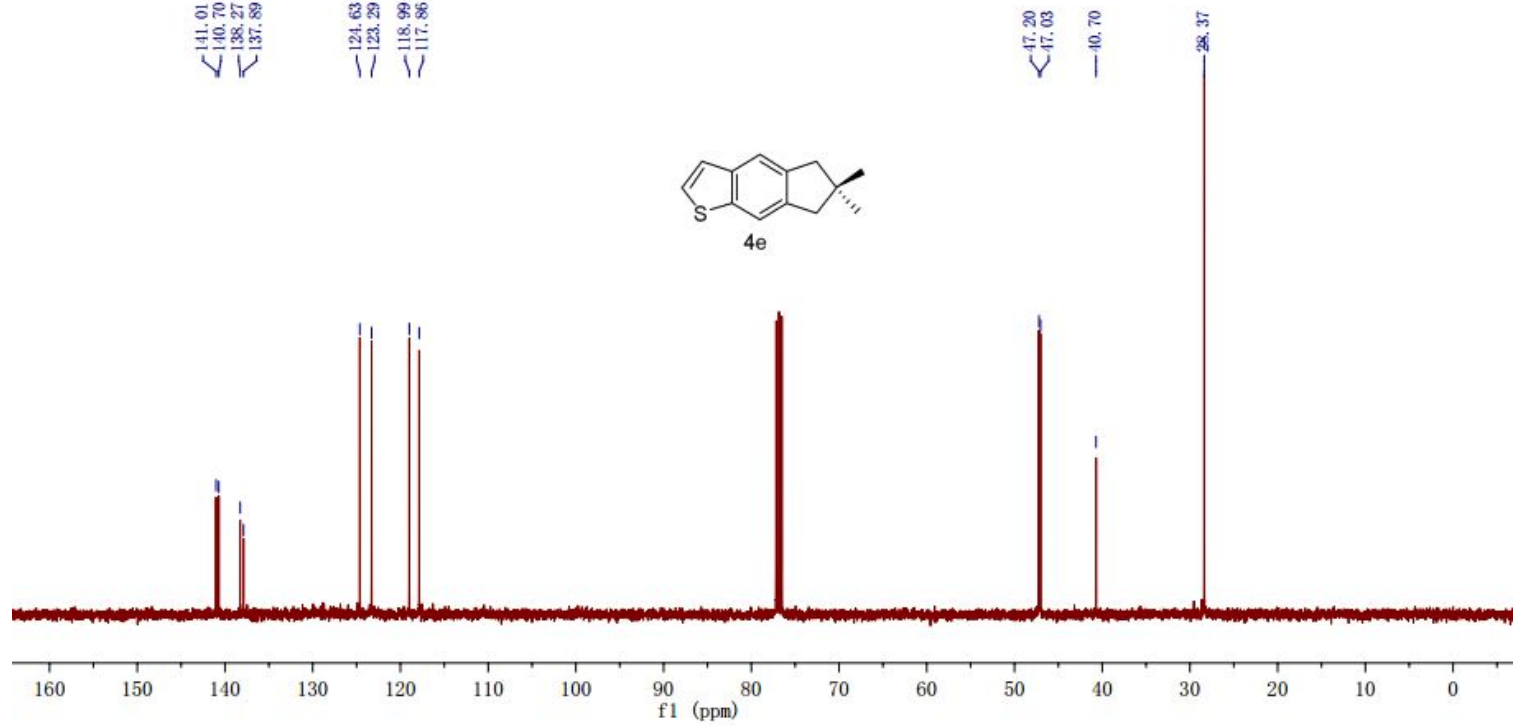
'H NMR $\left(400 \mathrm{MHz}_{\infty}, \mathrm{CDCl}_{3}\right)$ \%

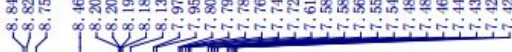

$\int S, \pi 5 \mid 1 /$

$88 \%$
พิنี

$\iint$

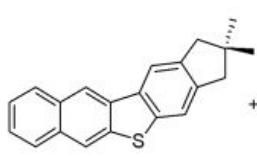

$4 \mathrm{fa}$

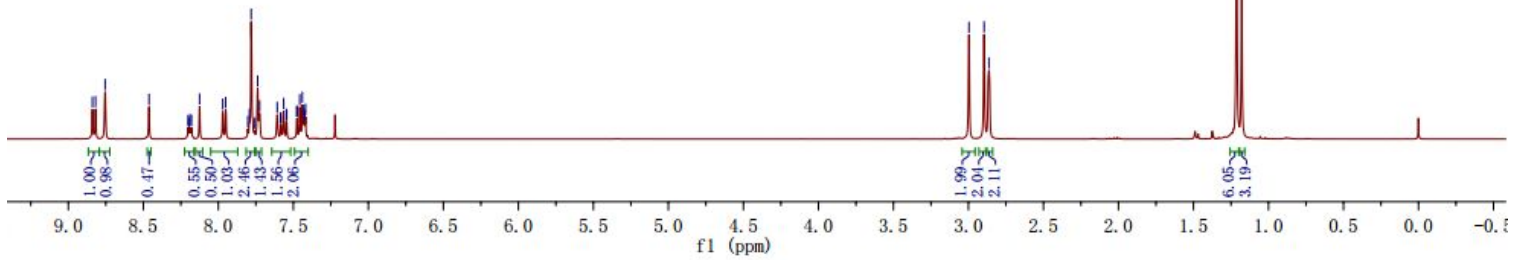

${ }^{13} \mathrm{C}\{\mathrm{lH}\} \mathrm{NMR}(100 \mathrm{MHz}, \mathrm{CDCl})$

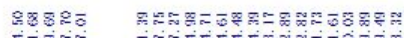

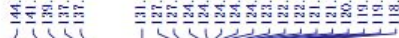

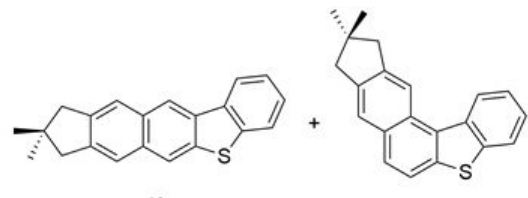

4 fa

$4 \mathrm{fb}$

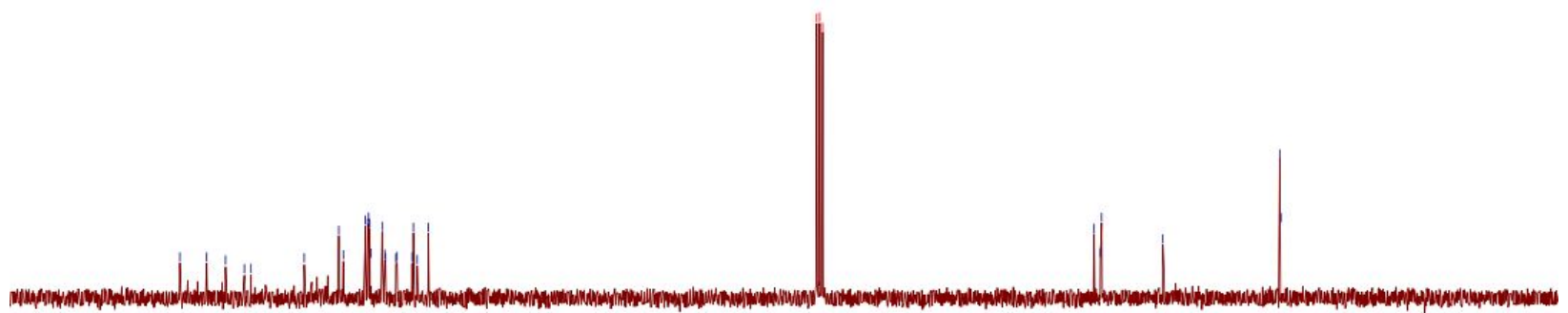

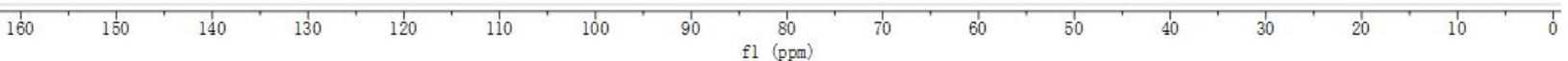


'H NMR (400 MHz, $\left.\mathrm{CDCl}_{3}\right)$

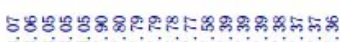

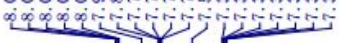

$\iiint \int \mid$
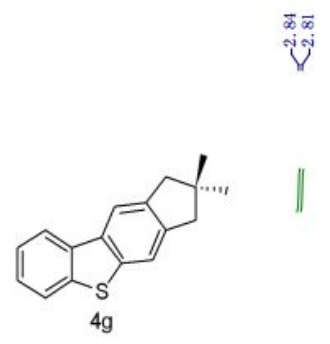

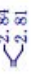

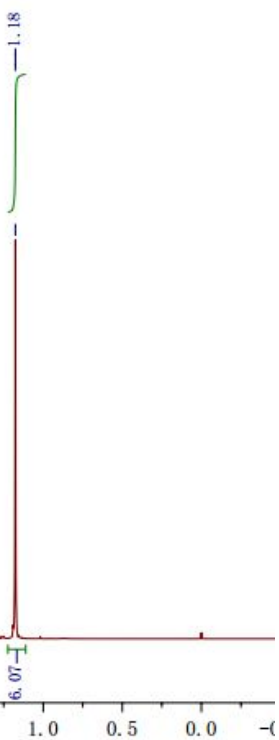

f1 (ppm)

${ }^{13} \mathrm{C}\{\mathrm{IH}\} \mathrm{NMR}\left(100 \mathrm{MHz}, \mathrm{CDCl}_{3}\right)$

\begin{tabular}{|c|c|}
\hline 구음유 & ஜ88\$8 \\
\hline 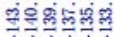 & 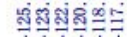 \\
\hline
\end{tabular}
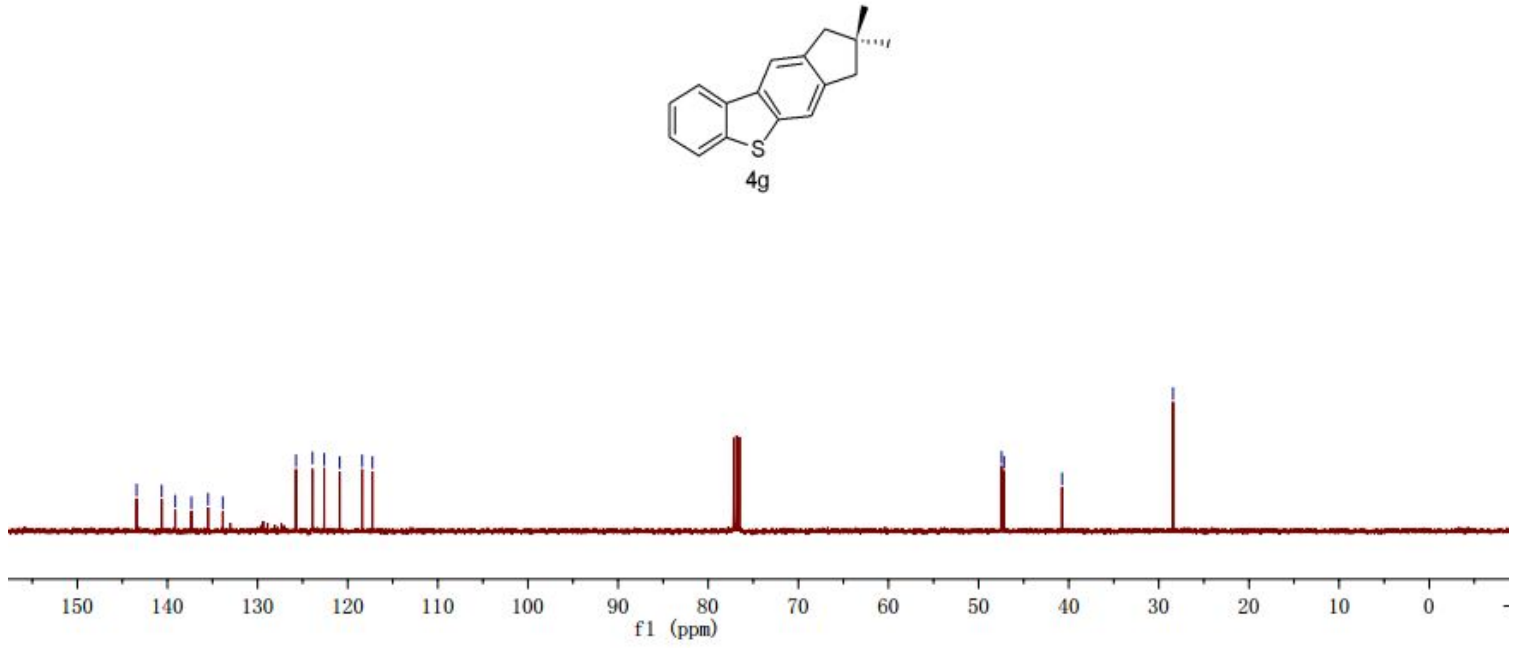


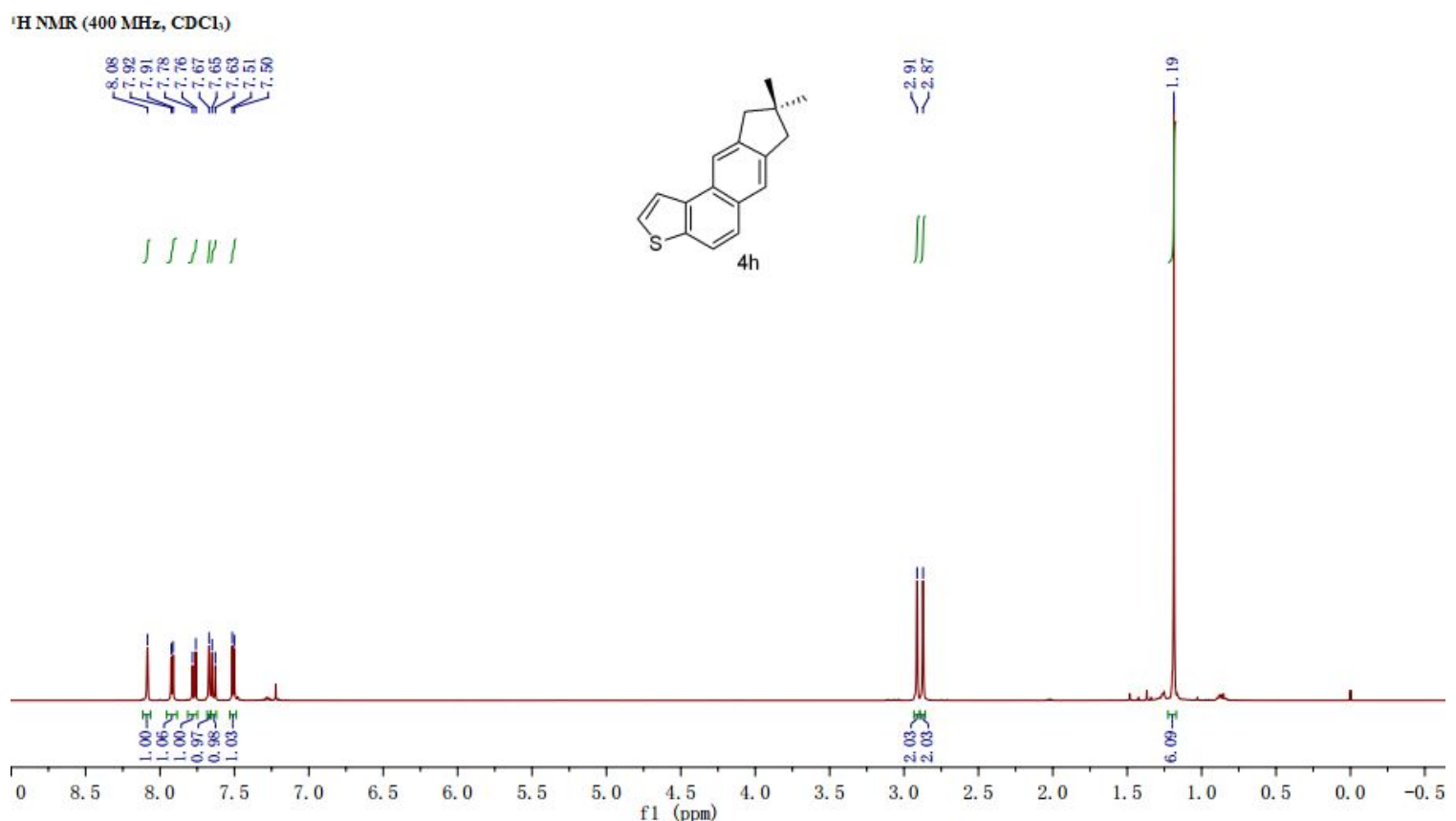

${ }_{13}^{13} \mathrm{C}\{1 \mathrm{H}\}$ NMR $\left(100 \mathrm{MHz}, \mathrm{CDCl}_{3}\right)$
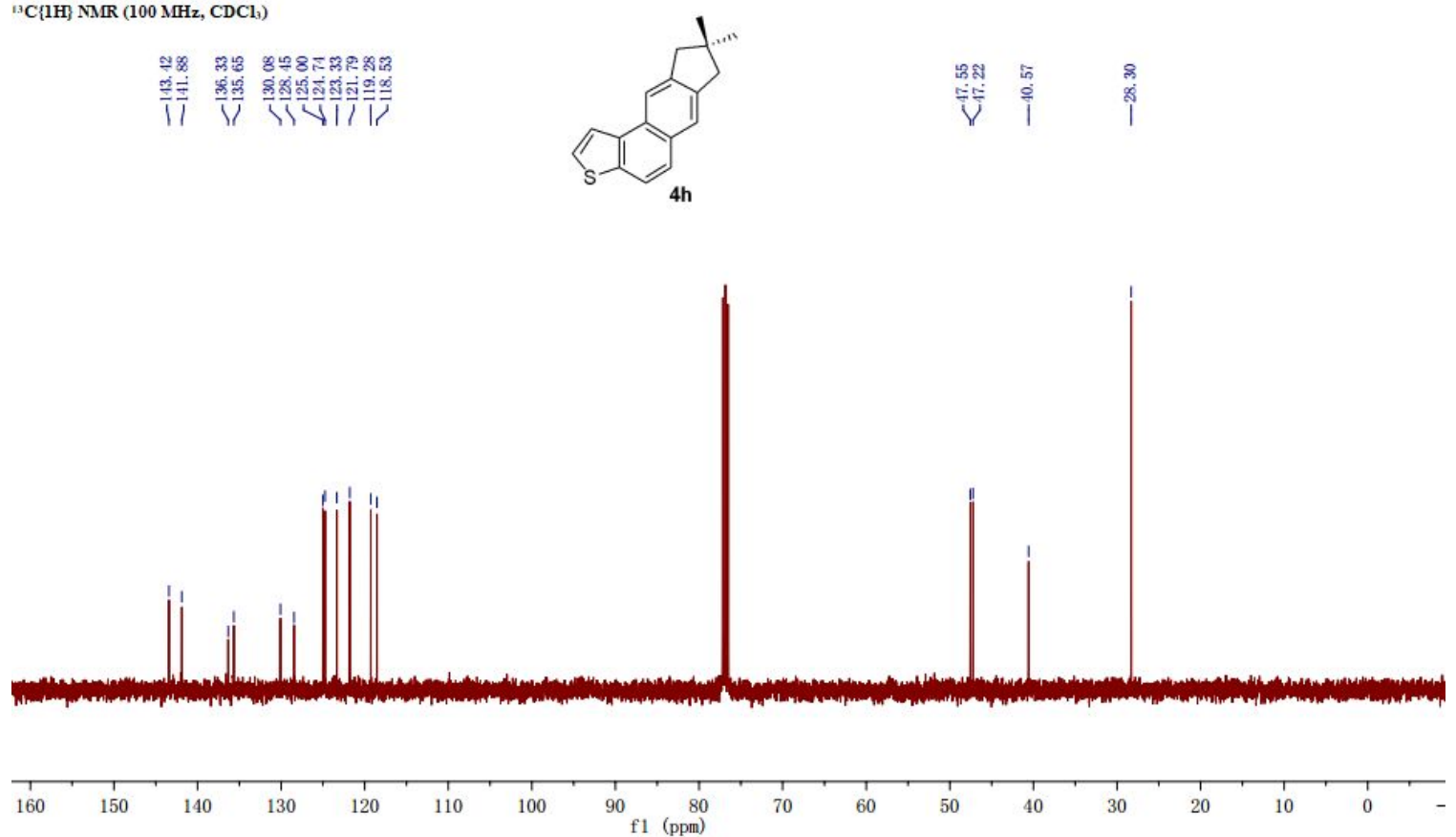
' $\mathrm{H}$ NMR $\left(400 \mathrm{MHz}, \mathrm{CDCl}_{3}\right)$

$\begin{array}{ll}108 \\ i & i\end{array}$

s S

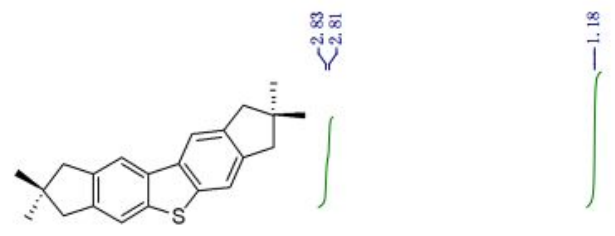

$4 i$

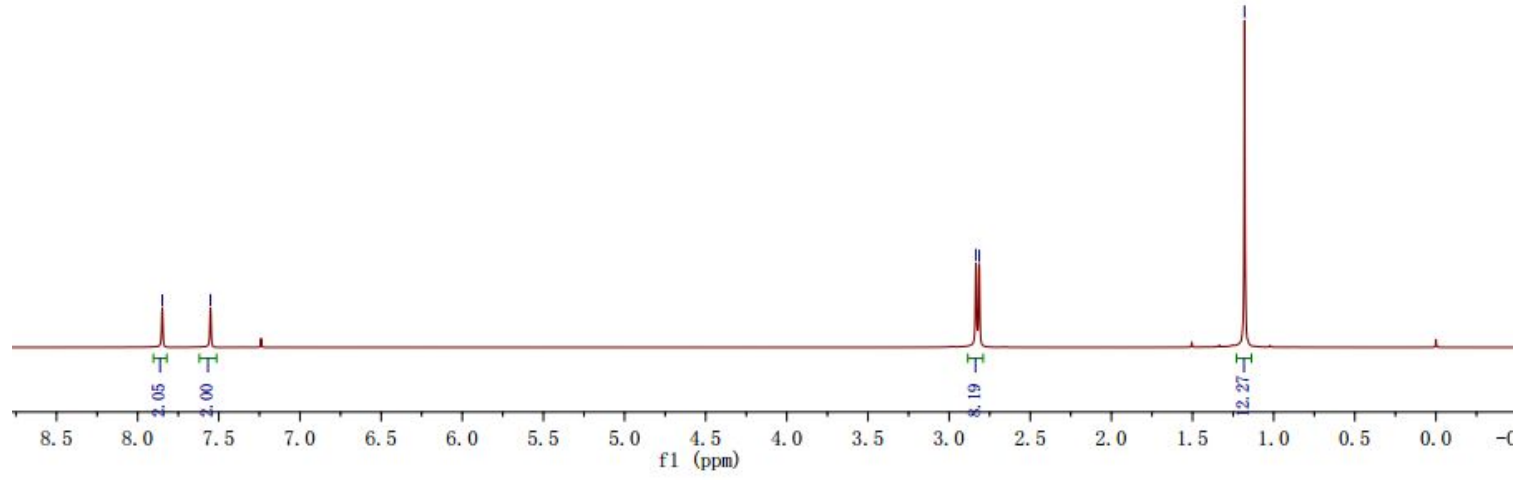

${ }^{13} \mathrm{C}\{1 \mathrm{H}\}$ NMR $\left(100 \mathrm{MHz}, \mathrm{CDCl}_{3}\right)$

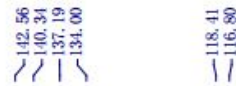

守
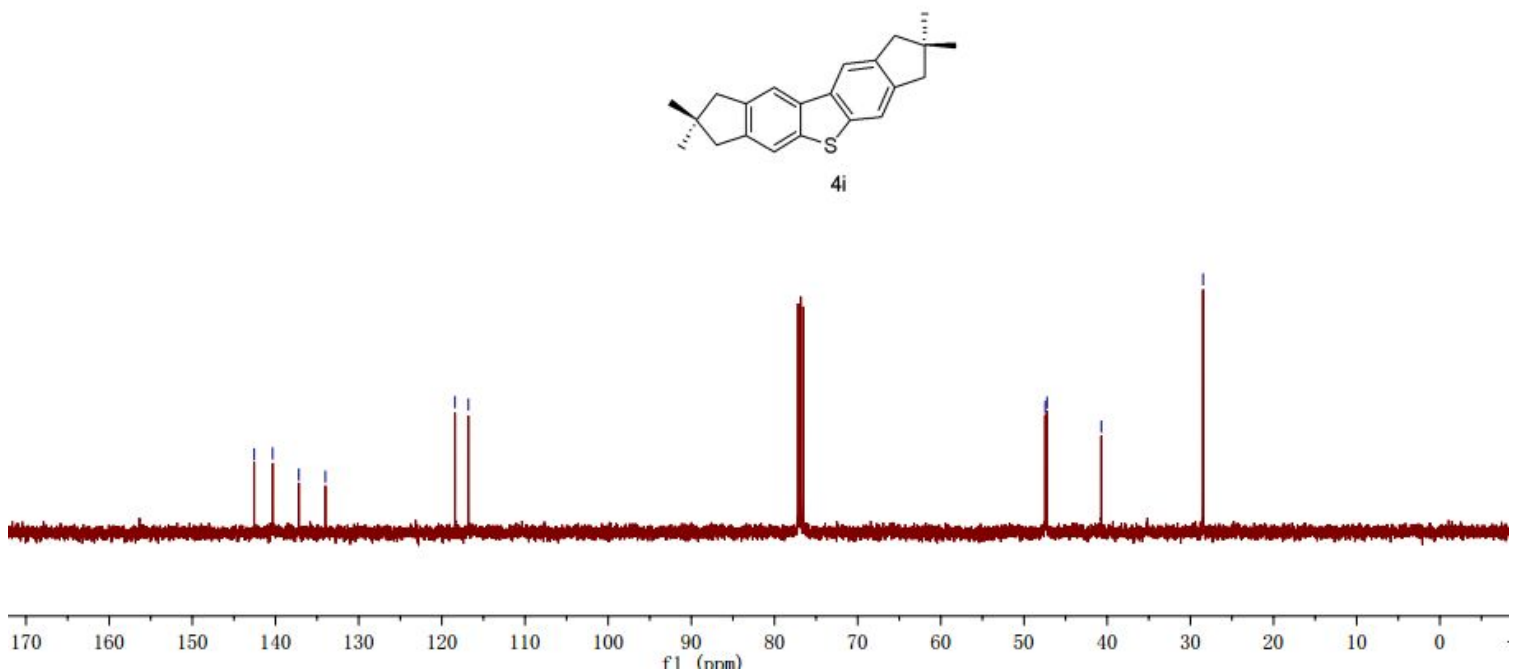
'H NMR (400 MHz, $\left.\mathrm{CDCl}_{3}\right)$

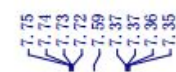

111

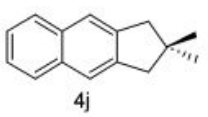

$\stackrel{\mathscr{\infty}}{\sim}$
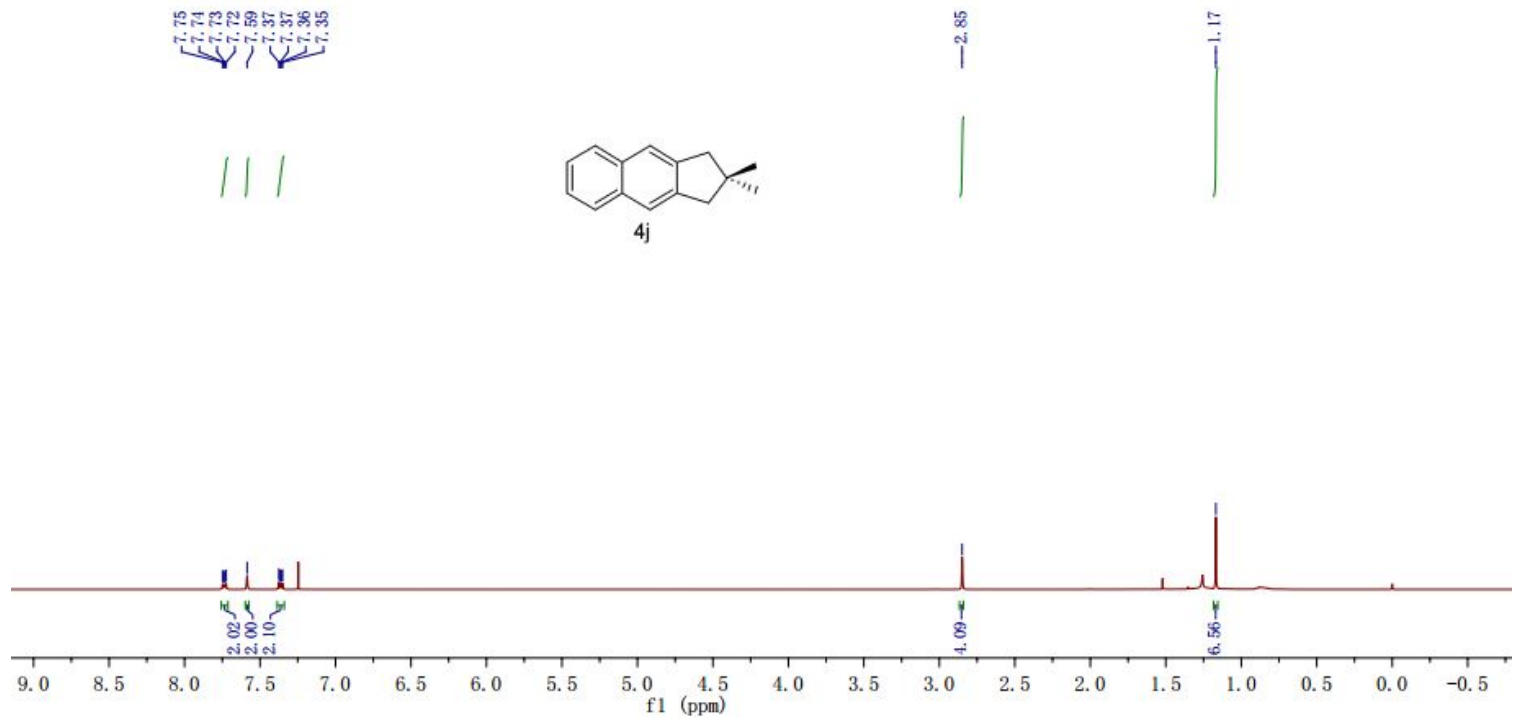

${ }^{13} \mathrm{C}\{1 \mathrm{H}\} \mathrm{NMR}\left(100 \mathrm{MHz}, \mathrm{CDCl}_{3}\right)$

$\begin{array}{lll}1 & 8 & \text { क⿱ के } \\ \frac{1}{2} & 11\end{array}$

욤
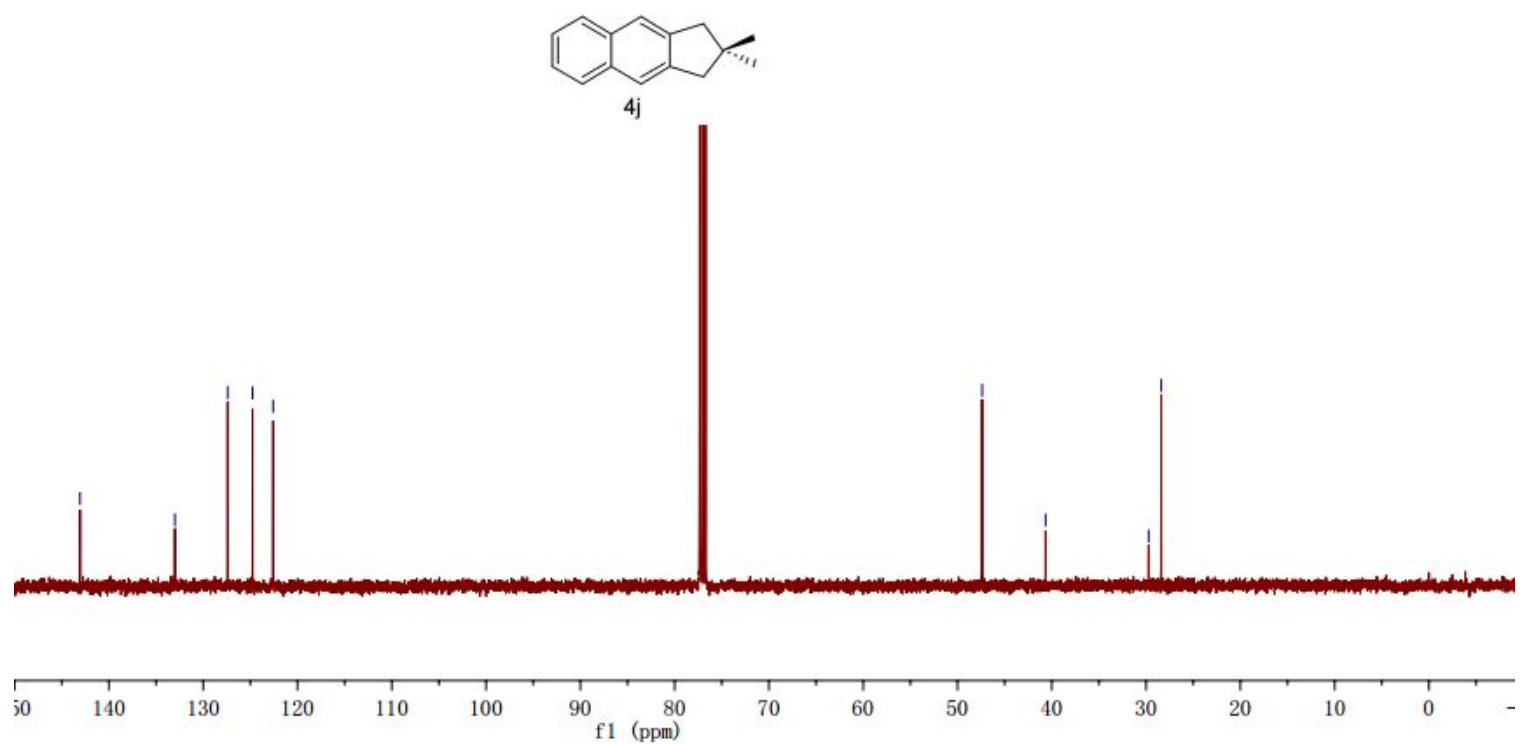


\section{Absorption and Emission Spectra}
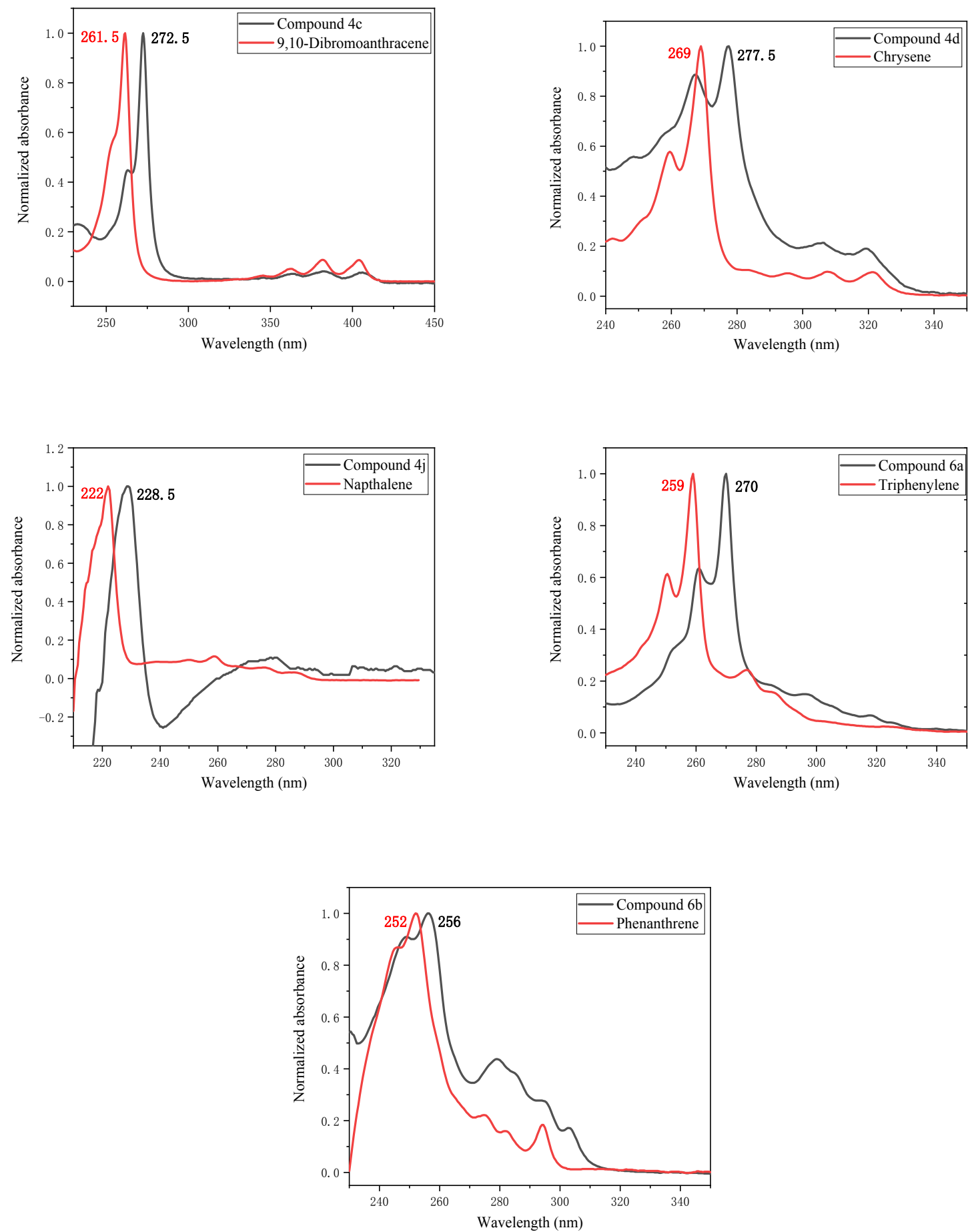

Figure S1. UV-vis absorption of $\mathbf{4 c}, \mathbf{4 d}, \mathbf{4 j}, \mathbf{6 a}, \mathbf{6 b}$ and their unsubstituted parent compounds in solution (THF, $0.01 \mathrm{mM}$ ), All compounds have a red shift. 

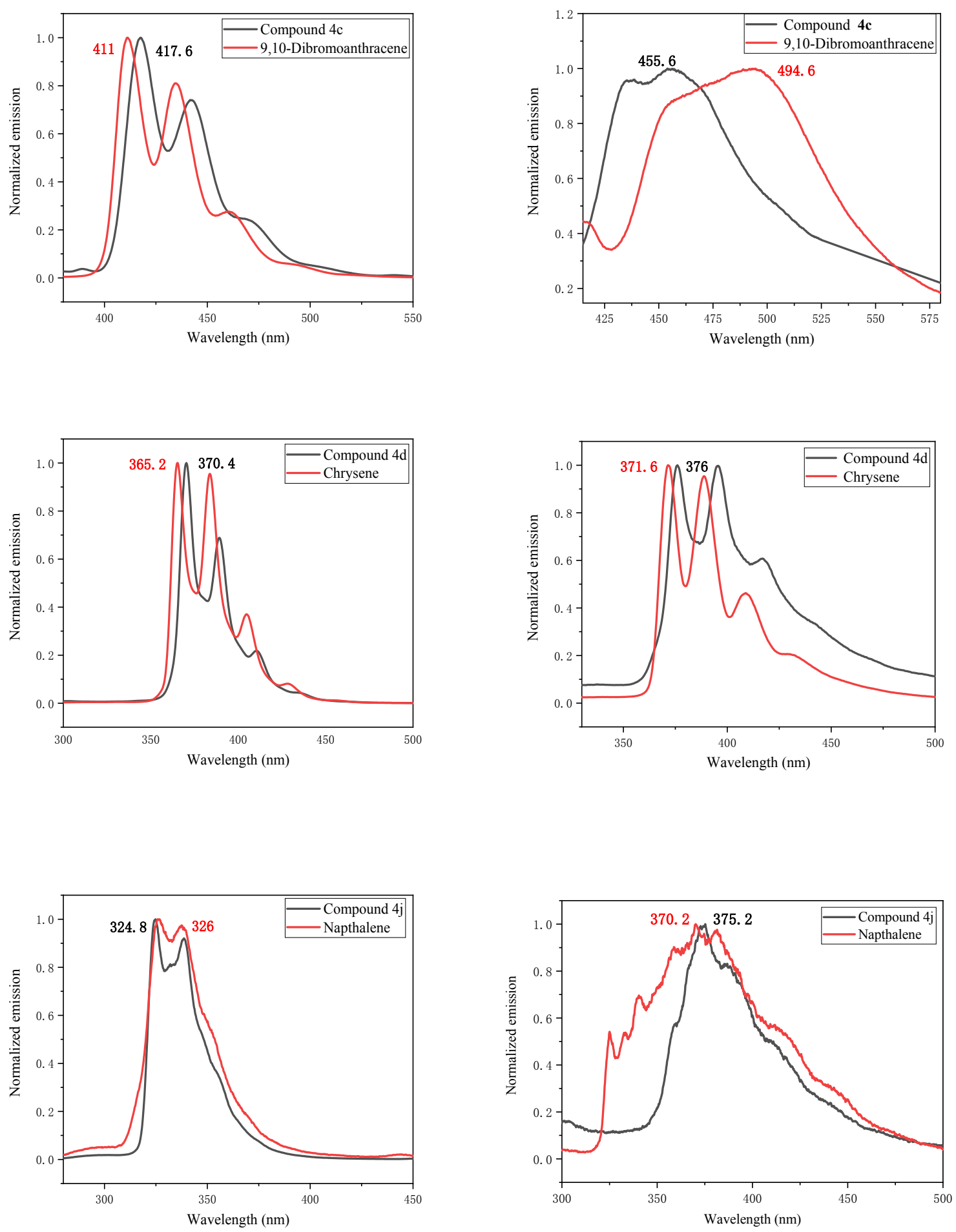

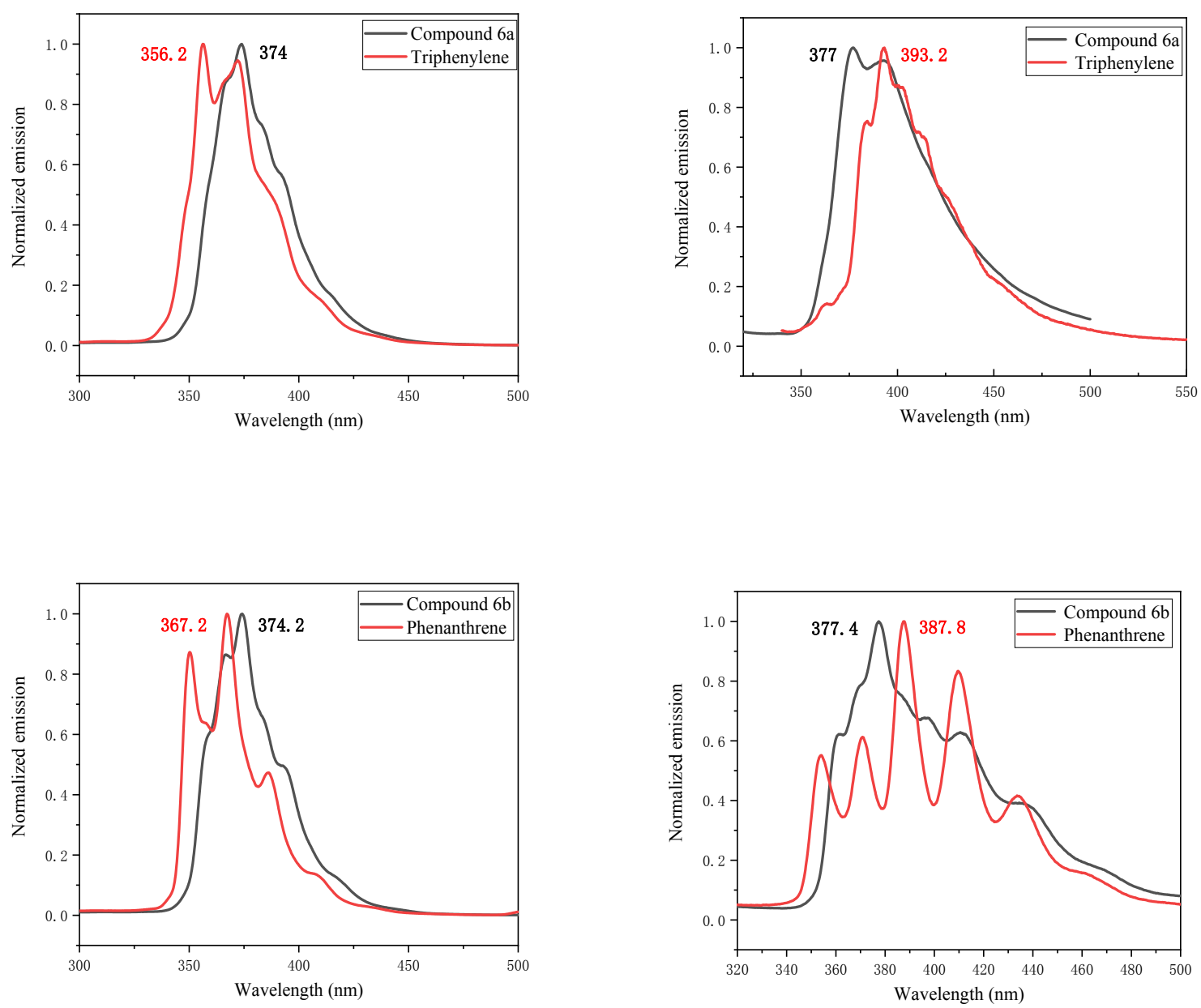

Figure S2. Fluorescence emission spectra of $\mathbf{4 c}, \mathbf{4 d}, \mathbf{4 j}, \mathbf{6 a}, \mathbf{6 b}$ and their unsubstituted parent compounds in solution (THF, $0.01 \mathrm{mM}$, left) and thin film (right).

Excitation wavelength: $\mathbf{4 c}-278 \mathrm{~nm}$; Dibromoanthracene $-365 \mathrm{~nm} ; \mathbf{4 d}-277 \mathrm{~nm}$; Chrysene $-277 \mathrm{~nm}$; 4j-228 nm; Napthalene - $222 \mathrm{~nm} ; \mathbf{6 a}-280 \mathrm{~nm}$; Triphenylene -307 nm; 6b-260 nm; Phenanthrene$260 \mathrm{~nm}$; PMT Voltage: 4c (680V in thin film, 480V in solution), 4d (570V in thin film, 430V in solution), $4 \mathbf{j}(700 \mathrm{~V}$ in thin film, $650 \mathrm{~V}$ in solution), $6 \mathbf{a}(470 \mathrm{~V}$ in thin film, $470 \mathrm{~V}$ in solution), $6 \mathbf{b}(500 \mathrm{~V}$ in thin film, $480 \mathrm{~V}$ in solution). 

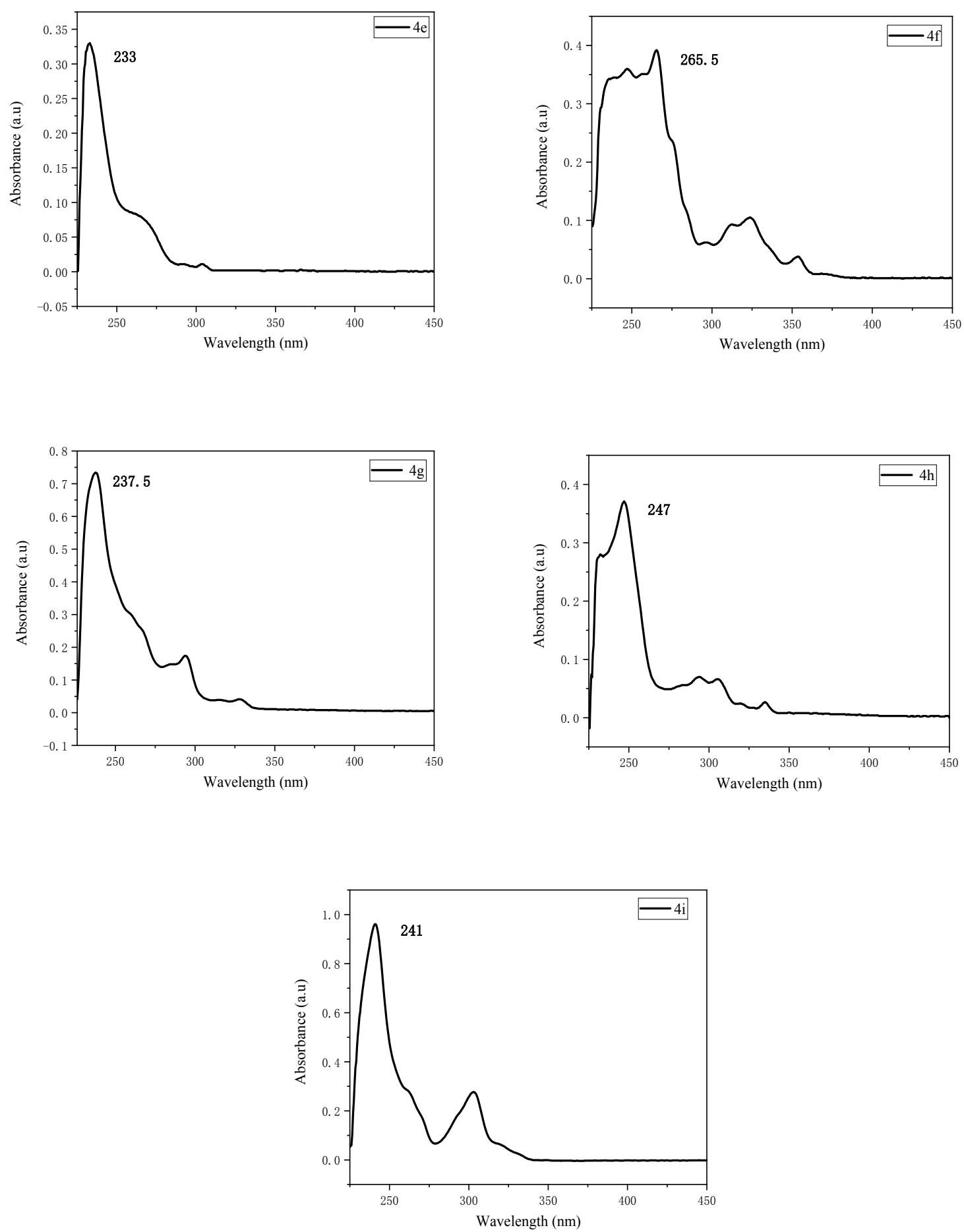

Figure S3. UV-vis absorption of compound 4e-4i in solution (THF, $0.01 \mathrm{mM}$ ). 

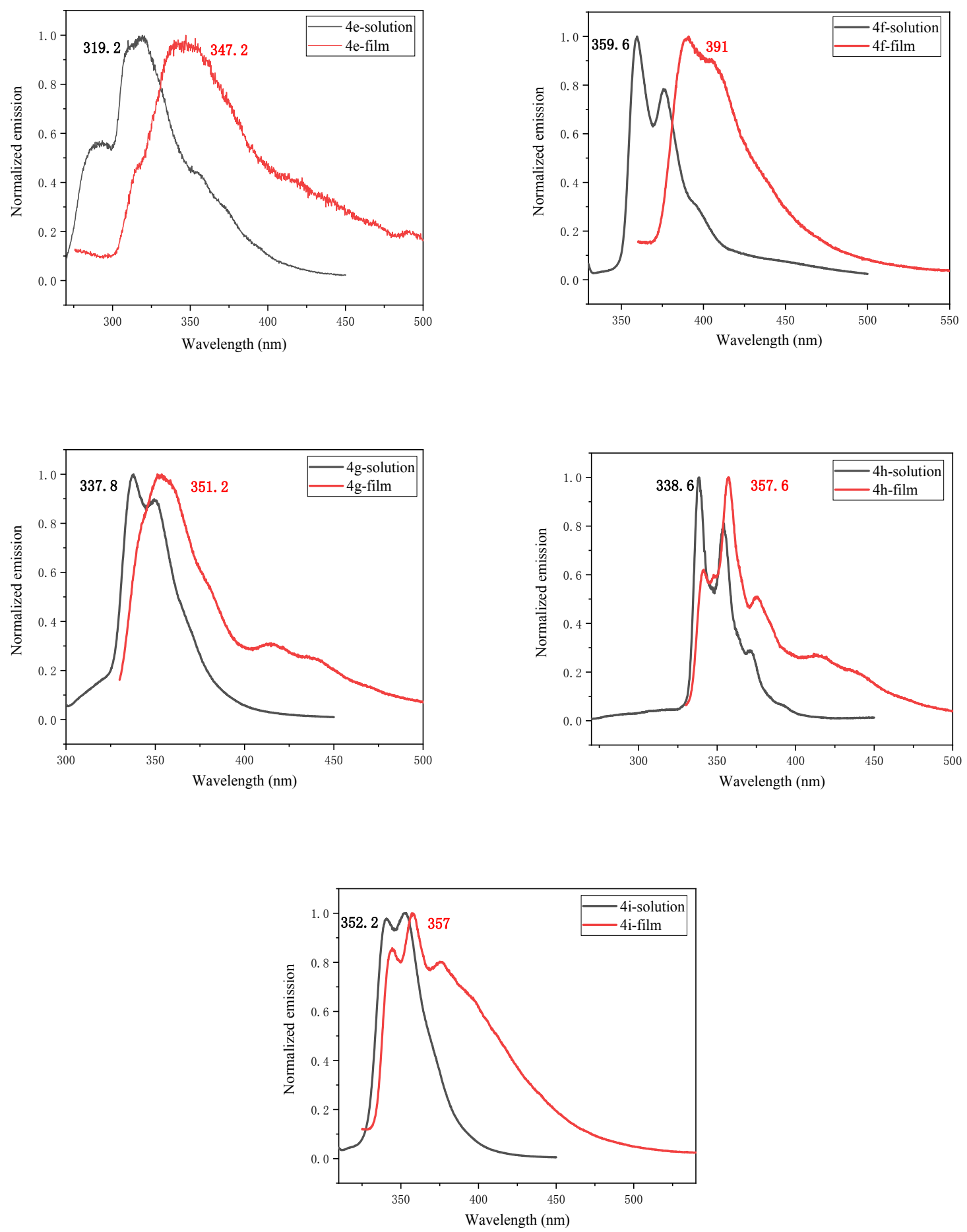

Figure S4. Fluorescence emission spectra of compound 4e-4i in solution (THF, $0.01 \mathrm{mM}$ ) and thin film. These compounds were excited at the laser setting of the maximum absorption wavelength, PMT Voltage: $4 \mathrm{e}(700 \mathrm{~V}$ in thin film, $720 \mathrm{~V}$ in solution), $4 \mathrm{f}(700 \mathrm{~V}$ in thin film, $720 \mathrm{~V}$ in solution), $4 \mathrm{~g}(600 \mathrm{~V}$ in thin film, $600 \mathrm{~V}$ in solution), $4 \mathrm{~h}(600 \mathrm{~V}$ in thin film, $580 \mathrm{~V}$ in solution), $4 \mathbf{i}(600 \mathrm{~V}$ in thin film, $720 \mathrm{~V}$ in solution). 


\section{Oak Ridge Thermal Ellipsoid Plot diagram of compound 4c}

The crystal sample of $4 \mathrm{c}$ was obtained via solvent evaperation of its DCM solution at room temperature.

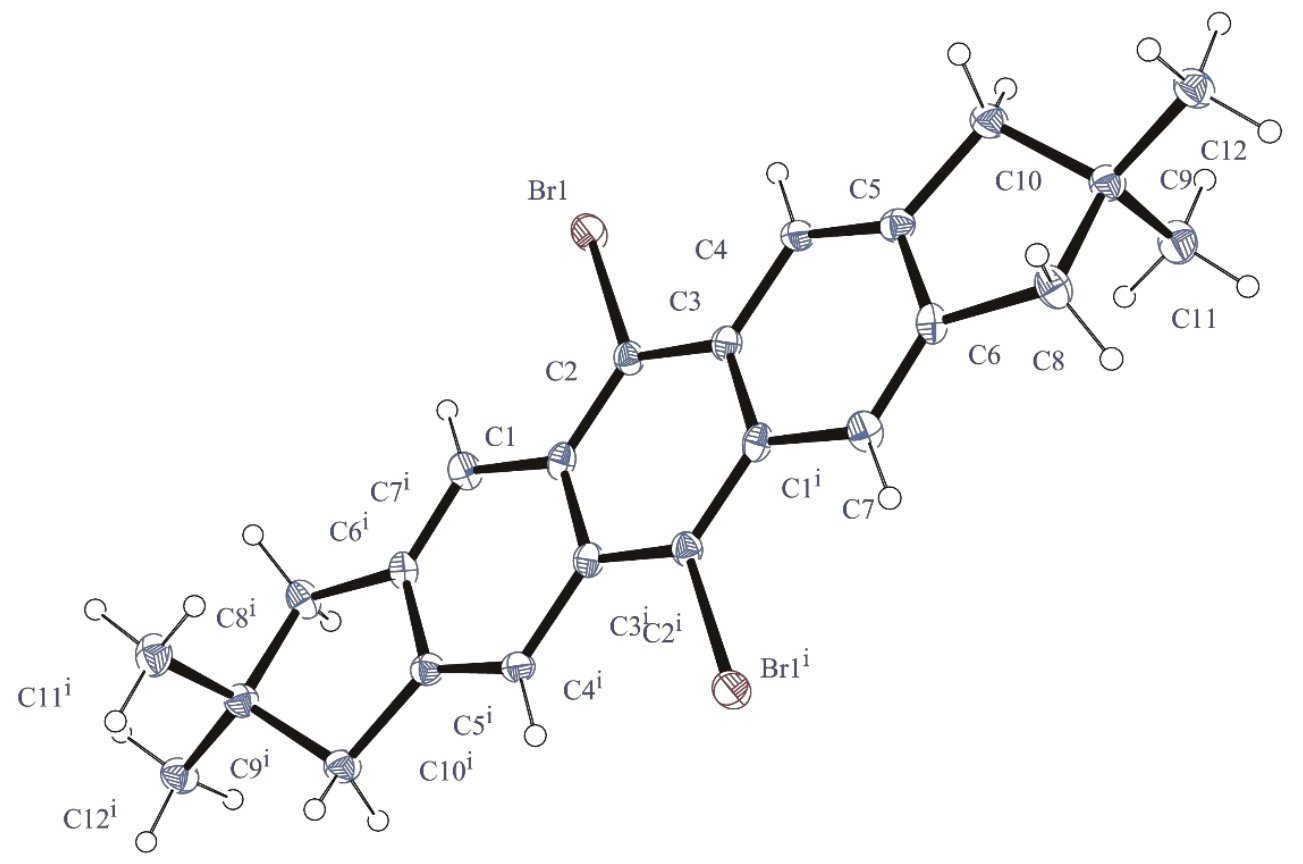

Figure S5. The ORTEP diagram of compound 4c. The thermal ellipsoid is set at the $30 \%$ probability level.

Table S1. Crystallographic data for compound 4c

\begin{tabular}{|c|c|}
\hline T(K) & 160.00000 \\
\hline Formula & C12 H12 Br \\
\hline Formula weight & 236.13000 \\
\hline System & monoclinic \\
\hline Space group & P 2 $1 /$ n \\
\hline a $(\AA)$ & $6.23670(10)$ \\
\hline b $(\AA)$ & $20.0259(4)$ \\
\hline c $(\AA)$ & $8.06700(10)$ \\
\hline$\alpha^{\circledR}\left(^{\circ}\right)$ & 90 \\
\hline$\beta\left(^{\circ}\right)$ & $96.856(2)$ \\
\hline$\gamma\left(^{\circ}\right)$ & 90 \\
\hline V $(\AA 3)$ & $1000.33(3)$ \\
\hline$Z$ & 4 \\
\hline F $(000)$ & 476 \\
\hline
\end{tabular}




\section{Frontier molecular orbitals}
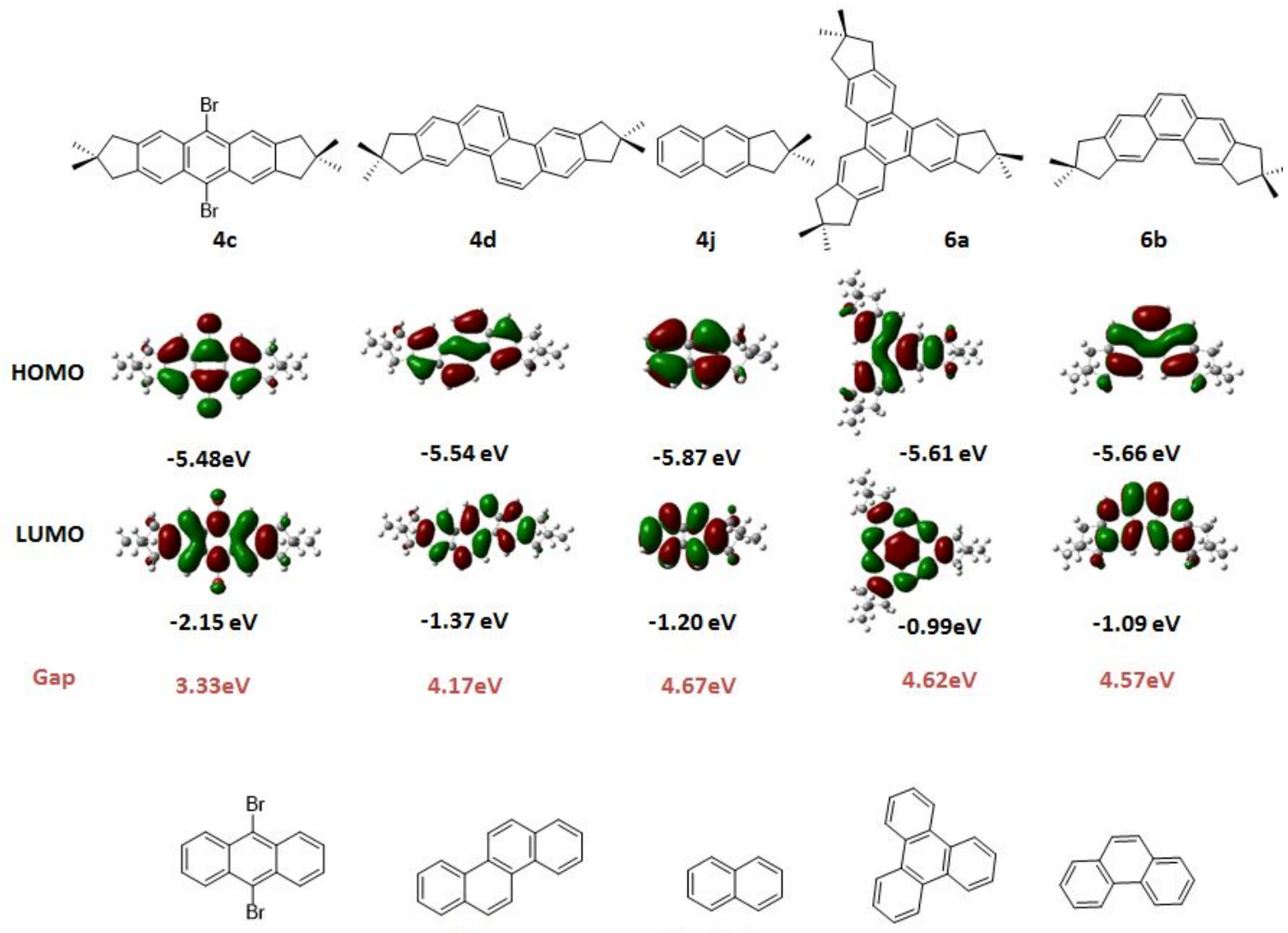

9,10-Dibromoanthracene

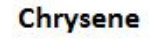

Napthalene

Triphenylene

Phenanthrene

номо
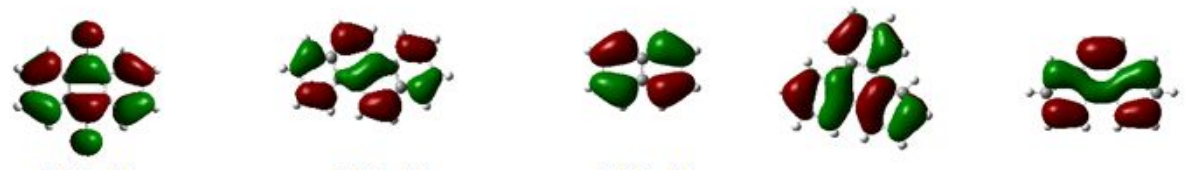

$-5.81 \mathrm{eV}$

$-6.09 \mathrm{eV}$

$-6.15 \mathrm{eV}$

$-6.02 \mathrm{eV}$

LUMO
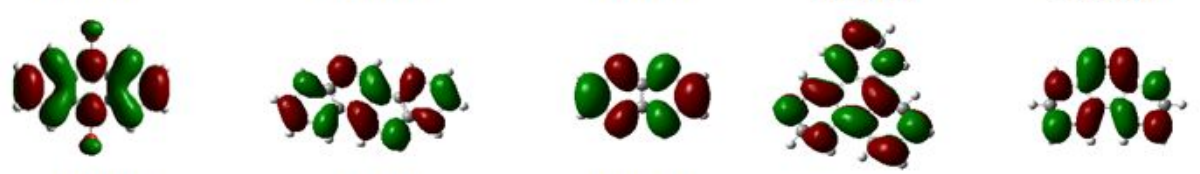

$-2.49 \mathrm{eV}$

$-1.61 \mathrm{eV}$

$-1.35 \mathrm{eV}$

$-1.31 \mathrm{eV}$

$-1.36 \mathrm{eV}$

$4.74 \mathrm{eV}$

$4.84 \mathrm{eV}$

$4.67 \mathrm{eV}$

Figure S6. Plots of calculated molecular frontier orbitals and their corresponding energies for the gemdimethylcyclopentane-fused arenes $\mathbf{4 c}, \mathbf{4 d}, \mathbf{4 j}, \mathbf{6 a}$ and $\mathbf{6 b}$ (on the top) and their unsubstituted parent compounds 9,10-dibromoanthracene, chrysene, naphthalene, triphenylene, phenanthrene (on the bottom). 

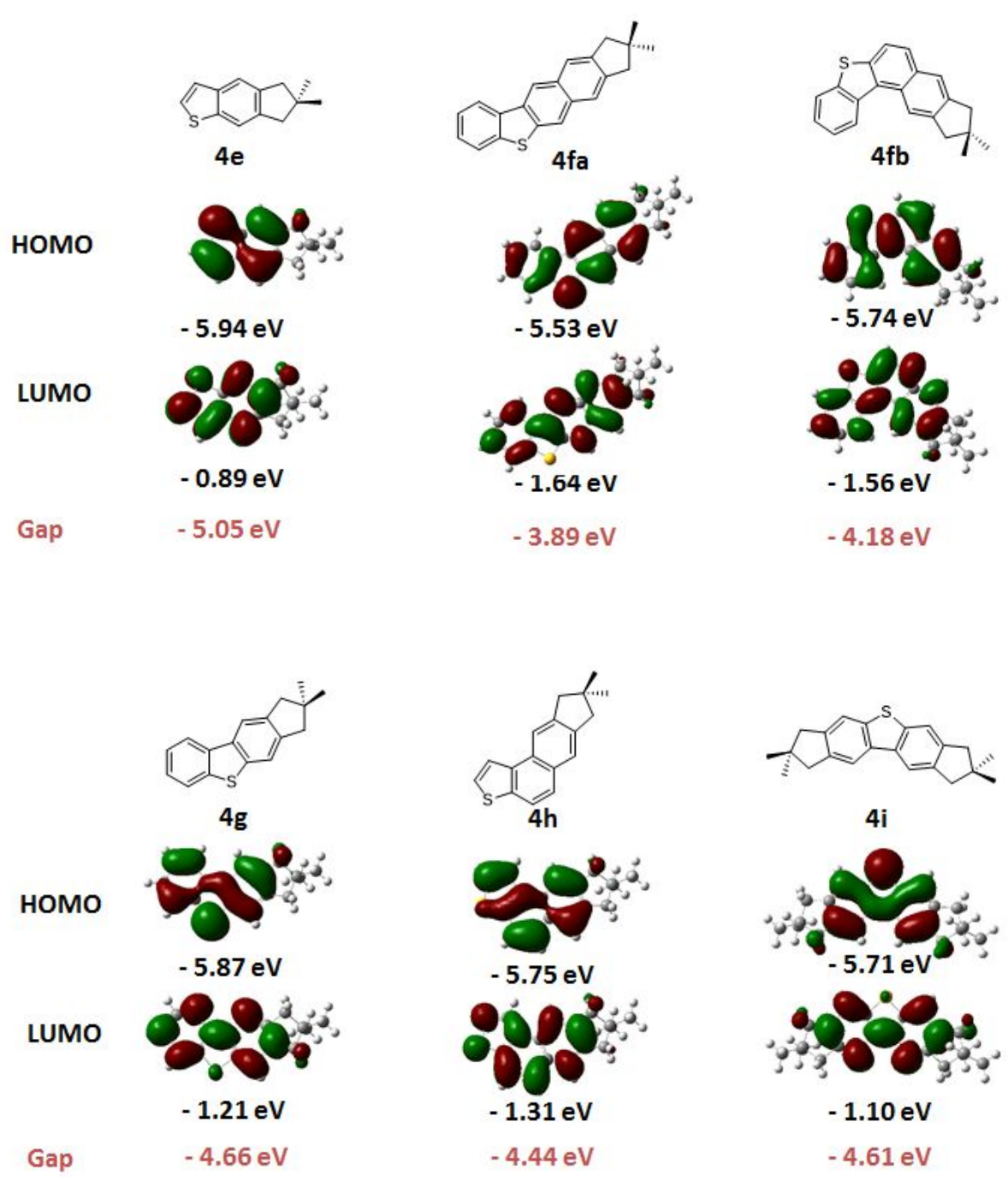

Figure S7. Plots of calculated molecular frontier orbitals and their corresponding energies for the gemdimethylcyclopentane-fused arenes $\mathbf{4 e - 4 i}$. 


\section{Cyclic voltammetry curves}
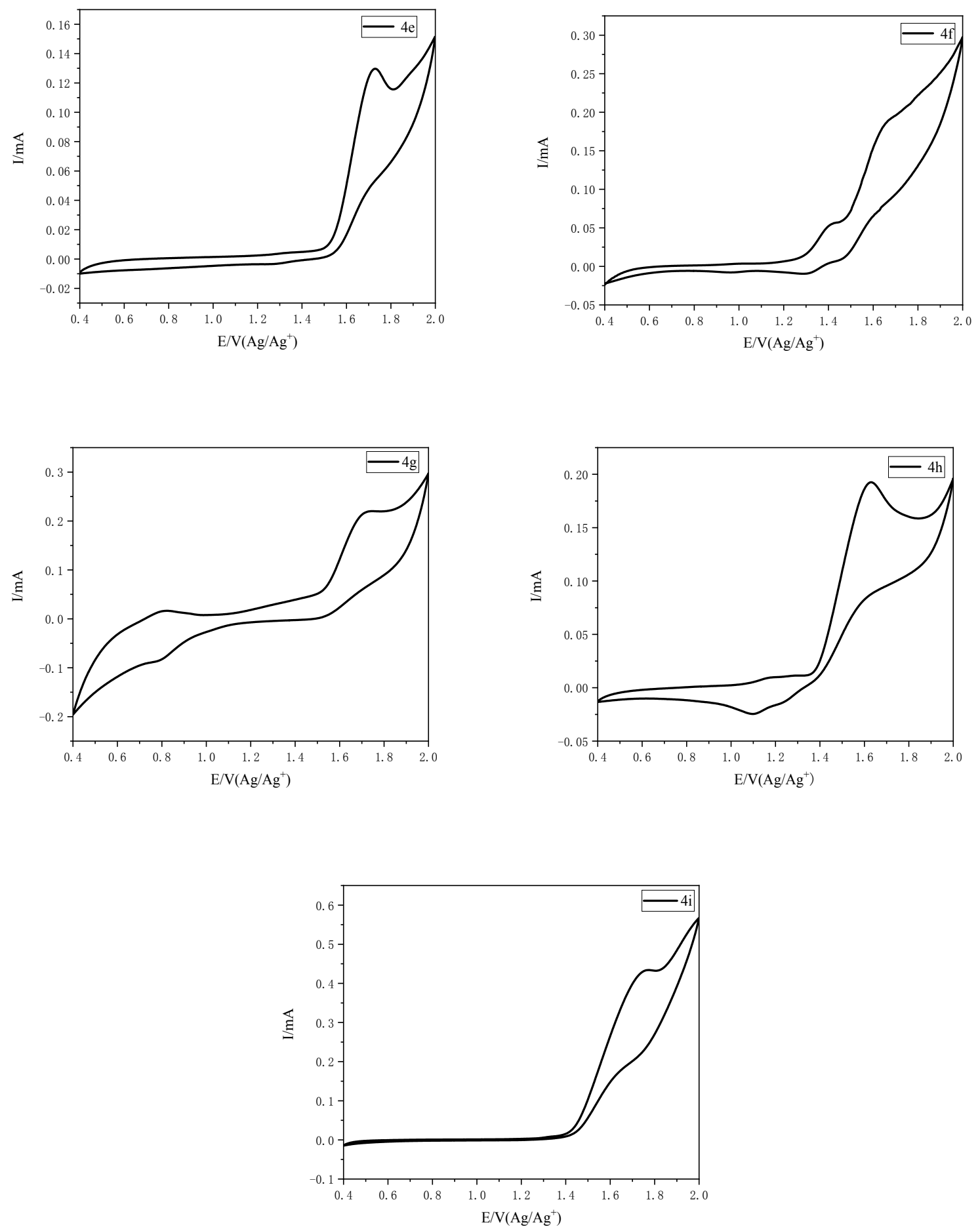

Figure S8. Cyclic voltammograms of $4 \mathbf{e}-4 \mathbf{i}$ at a scan rate of $50 \mathrm{mV} / \mathrm{s}$. 


\section{Computational calculation}

All calculations were performed using density functional theory (DFT) by Gaussian16 soft-ware package, with B3LYP exchange-correlation functional. The basis set of 6-31+G (d, p) for all atoms was used for structural optimizations and frequency calculations. 0 imaginary frequency was obtained for all of the calculations.

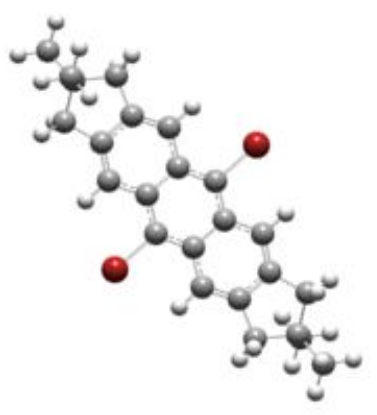

Total Energy $=-6072.222549$ Hartree HOMO: -0.21339 Hartree $=-5.4771009 \mathrm{eV}$ LUMO: -0.07919 Hartree $=-2.1547599 \mathrm{eV}$

Cartesian coordinates:

$\begin{array}{lrrr}\text { Atoms } & \mathbf{X} & \mathbf{Y} & \mathbf{Z} \\ \mathrm{C} & 3.66177630 & -0.71422809 & -0.26365916 \\ \mathrm{C} & 2.49076895 & -1.41289094 & -0.17718580 \\ \mathrm{C} & 1.23496402 & -0.72690121 & -0.08958945 \\ \mathrm{C} & 1.23493998 & 0.72695154 & -0.08954603 \\ \mathrm{C} & 2.49074747 & 1.41296733 & -0.17695490 \\ \mathrm{C} & 3.66176602 & 0.71433253 & -0.26352497 \\ \mathrm{C} & 0.00004612 & -1.39158360 & -0.00046962 \\ \mathrm{C} & -0.00000522 & 1.39157725 & -0.00023687 \\ \mathrm{C} & -1.23489611 & 0.72688300 & 0.08915200 \\ \mathrm{C} & -1.23487082 & -0.72694852 & 0.08910592 \\ \mathrm{C} & -2.49064449 & -1.41298354 & 0.17661422 \\ \mathrm{H} & -2.49202157 & -2.49600375 & 0.16743413 \\ \mathrm{C} & -3.66164578 & -0.71432946 & 0.26329988 \\ \mathrm{C} & -3.66167486 & 0.71426058 & 0.26344460 \\ \mathrm{C} & -2.49066088 & 1.41290596 & 0.17684854 \\ \mathrm{H} & 2.49215755 & -2.49591233 & -0.16814508 \\ \mathrm{H} & 2.49212943 & 2.49598461 & -0.16775212 \\ \mathrm{H} & -2.49201560 & 2.49593096 & 0.16781197 \\ \mathrm{Br} & 0.00006074 & -3.31538060 & 0.00006811 \\ \mathrm{Br} & -0.00019690 & 3.31537384 & -0.00001684 \\ \mathrm{C} & 5.08200519 & -1.21991029 & -0.36743276 \\ \mathrm{H} & 5.27160331 & -2.11128826 & 0.24104711 \\ \mathrm{H} & 5.30559185 & -1.48986426 & -1.41002778 \\ \mathrm{C} & 5.08198169 & 1.22004976 & -0.36729199 \\ \mathrm{H} & 5.30554639 & 1.49011950 & -1.40986252 \\ \mathrm{H} & 5.27157541 & 2.11136478 & 0.24128057 \\ \mathrm{C} & 5.95499462 & 0.00005385 & 0.06683003 \\ \mathrm{C} & 7.33391089 & 0.00010616 & -0.60430815\end{array}$

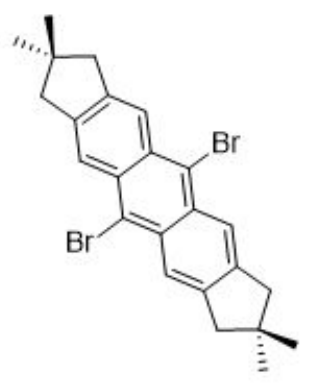




$\begin{array}{lrrr}\mathrm{H} & 7.24537546 & 0.00016194 & -1.69694110 \\ \mathrm{H} & 7.91347285 & 0.88448444 & -0.31416464 \\ \mathrm{H} & 7.91349518 & -0.88428754 & -0.31425647 \\ \mathrm{C} & 6.11820172 & -0.00003048 & 1.59974428 \\ \mathrm{H} & 6.67123910 & -0.88600412 & 1.93266950 \\ \mathrm{H} & 6.67122232 & 0.88591621 & 1.93276911 \\ \mathrm{H} & 5.14734111 & -0.00006822 & 2.10742005 \\ \mathrm{C} & -5.08188752 & 1.21987190 & 0.36772812 \\ \mathrm{H} & -5.30520719 & 1.48934172 & 1.41052387 \\ \mathrm{H} & -5.27175673 & 2.11148829 & -0.24029872 \\ \mathrm{C} & -5.08184279 & -1.22000134 & 0.36752817 \\ \mathrm{H} & -5.27164551 & -2.11153374 & -0.24066027 \\ \mathrm{H} & -5.30506426 & -1.48969176 & 1.41029439 \\ \mathrm{C} & -5.95486550 & -0.00011088 & -0.06651932 \\ \mathrm{C} & -7.33372710 & -0.00005944 & 0.60471566 \\ \mathrm{H} & -7.91319662 & -0.88465971 & 0.31504819 \\ \mathrm{H} & -7.24520792 & 0.00045127 & 1.69735039 \\ \mathrm{H} & -7.91351455 & 0.88409008 & 0.31423131 \\ \mathrm{C} & -6.11855220 & 0.00003456 & -1.59932810 \\ \mathrm{H} & -6.67178526 & -0.88588690 & -1.93205811 \\ \mathrm{H} & -6.67168244 & 0.88602847 & -1.93198985 \\ \mathrm{H} & -5.14786494 & -0.00002499 & -2.10730414\end{array}$

Figure S9. Cartesian coordinates of the S0 optimized structure of compound 4c.
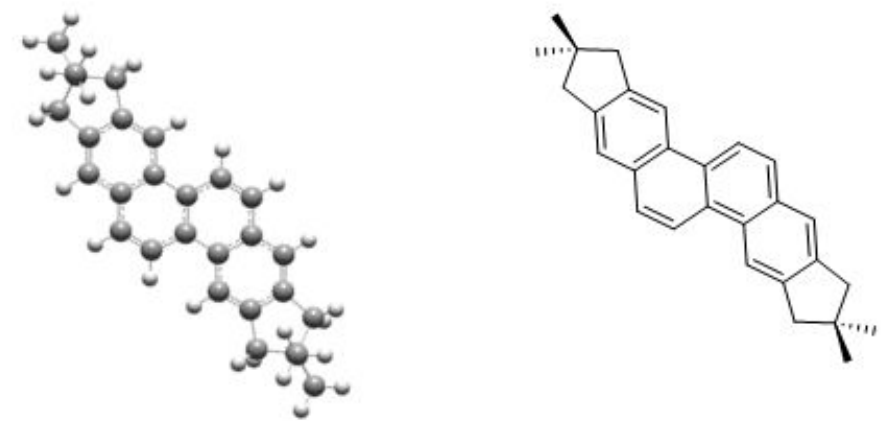

Total Energy $=-1083.560107$ Hartree

HOMO: -0.20345 Hartree $=-5.5358745 \mathrm{eV}$

LUMO: -0.05045 Hartree $=-1.3727445 \mathrm{eV}$

Cartesian coordinates:

$\begin{array}{lrrr}\text { Atoms } & \mathbf{X} & \mathbf{Y} & \mathbf{Z} \\ \mathrm{C} & -0.52298190 & 0.47994869 & -0.19951935 \\ \mathrm{C} & 0.52296122 & -0.47998532 & -0.19951267 \\ \mathrm{C} & 0.17675839 & -1.86922186 & -0.20041864 \\ \mathrm{C} & -1.12416814 & -2.28557611 & -0.20129488 \\ \mathrm{C} & -0.17677636 & 1.86918677 & -0.20039150 \\ \mathrm{C} & 1.91111365 & -0.04656080 & -0.20084464 \\ \mathrm{C} & 2.20508927 & 1.35305368 & -0.20266666 \\ \mathrm{C} & 1.12414776 & 2.28554067 & -0.20128068 \\ \mathrm{C} & 3.55261302 & 1.80284618 & -0.20007127\end{array}$




\begin{tabular}{|c|c|c|c|}
\hline $\mathrm{H}$ & 3.74574813 & 2.87364142 & -0.19508017 \\
\hline $\mathrm{C}$ & 4.58586987 & 0.89324908 & -0.19826326 \\
\hline $\mathrm{C}$ & 4.30419355 & -0.49535370 & -0.19545774 \\
\hline $\mathrm{C}$ & 3.00448815 & -0.95661001 & -0.19482967 \\
\hline $\mathrm{H}$ & -0.95910833 & 2.61829958 & -0.20148453 \\
\hline $\mathrm{H}$ & 0.95909644 & -2.61832796 & -0.20156749 \\
\hline $\mathrm{H}$ & -1.35504270 & -3.34791966 & -0.20205474 \\
\hline $\mathrm{H}$ & 1.35501447 & 3.34788658 & -0.20203472 \\
\hline $\mathrm{H}$ & 2.82681973 & -2.02635151 & -0.18491940 \\
\hline $\mathrm{C}$ & -2.20509929 & -1.35308130 & -0.20268562 \\
\hline $\mathrm{C}$ & -1.91112943 & 0.04651521 & -0.20090381 \\
\hline $\mathrm{C}$ & -3.55264248 & -1.80287718 & -0.20008033 \\
\hline $\mathrm{C}$ & -4.58588167 & -0.89328223 & -0.19832554 \\
\hline $\mathrm{C}$ & -3.00451005 & 0.95658662 & -0.19499104 \\
\hline $\mathrm{C}$ & -4.30420583 & 0.49534491 & -0.19563770 \\
\hline $\mathrm{H}$ & -2.82680265 & 2.02632257 & -0.18517456 \\
\hline $\mathrm{H}$ & -3.74576991 & -2.87367232 & -0.19500592 \\
\hline $\mathrm{C}$ & 6.08129463 & 1.11626017 & -0.20647796 \\
\hline $\mathrm{H}$ & 6.42250425 & 1.34123281 & -1.22774388 \\
\hline $\mathrm{H}$ & 6.40004893 & 1.95250397 & 0.42681052 \\
\hline $\mathrm{C}$ & 5.59822914 & -1.27926222 & -0.20251300 \\
\hline $\mathrm{H}$ & 5.82515082 & -1.62120708 & -1.22317131 \\
\hline $\mathrm{H}$ & 5.56956488 & -2.17215576 & 0.43309503 \\
\hline $\mathrm{C}$ & 6.67356748 & -0.24895856 & 0.26931726 \\
\hline $\mathrm{C}$ & 8.05537771 & -0.52866952 & -0.33505715 \\
\hline $\mathrm{H}$ & 8.43479335 & -1.50686824 & -0.01622711 \\
\hline $\mathrm{H}$ & 8.01924871 & -0.52349823 & -1.43068288 \\
\hline $\mathrm{H}$ & 8.78445108 & 0.22721864 & -0.01950095 \\
\hline $\mathrm{C}$ & 6.76397307 & -0.26454904 & 1.80811358 \\
\hline $\mathrm{H}$ & 7.11536084 & -1.23852827 & 2.16839028 \\
\hline $\mathrm{H}$ & 7.46528639 & 0.49859650 & 2.16542710 \\
\hline $\mathrm{H}$ & 5.78929028 & -0.06748777 & 2.26792607 \\
\hline $\mathrm{C}$ & -5.59824518 & 1.27924666 & -0.20275462 \\
\hline $\mathrm{H}$ & -5.56942158 & 2.17226283 & 0.43266660 \\
\hline $\mathrm{H}$ & -5.82528005 & 1.62098921 & -1.22344896 \\
\hline $\mathrm{C}$ & -6.08132304 & -1.11621288 & -0.20651207 \\
\hline $\mathrm{H}$ & -6.42248457 & -1.34111419 & -1.22781517 \\
\hline $\mathrm{H}$ & -6.40022404 & -1.95244483 & 0.42671292 \\
\hline $\mathrm{C}$ & -6.67350281 & 0.24903364 & 0.26928604 \\
\hline $\mathrm{C}$ & -6.76373822 & 0.26461628 & 1.80808243 \\
\hline $\mathrm{H}$ & -5.78910332 & 0.06721477 & 2.26782193 \\
\hline $\mathrm{H}$ & -7.11482822 & 1.23866847 & 2.16840106 \\
\hline $\mathrm{H}$ & -7.46526046 & -0.49834938 & 2.16535098 \\
\hline $\mathrm{C}$ & -8.05547698 & 0.52873242 & -0.33463888 \\
\hline $\mathrm{H}$ & -8.78441218 & -0.22718509 & -0.01886201 \\
\hline $\mathrm{H}$ & -8.43481446 & 1.50688009 & -0.01558232 \\
\hline $\mathrm{H}$ & -8.01979890 & 0.52363140 & -1.43027021 \\
\hline
\end{tabular}

Figure S10. Cartesian coordinates of the S0 optimized structure of compound $\mathbf{4 d}$. 

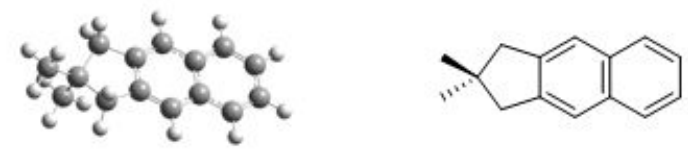

Total Energy $=-581.07313$ Hartree

HOMO: -0.21577 Hartree $=-5.8711017 \mathrm{eV}$

LUMO: -0.04404 Hartree $=-1.1983284 \mathrm{eV}$

Cartesian coordinates:

$\begin{array}{lrrr}\text { Atoms } & \mathbf{X} & \mathbf{Y} & \mathbf{Z} \\ \mathrm{C} & -4.92534269 & -0.24871149 & 0.16612371 \\ \mathrm{C} & -3.73398284 & 0.06532742 & 0.83459260 \\ \mathrm{C} & -3.16483527 & 1.33801133 & 0.69533303 \\ \mathrm{C} & -3.80044123 & 2.30849752 & -0.12622138 \\ \mathrm{C} & -4.99096721 & 1.98457606 & -0.79012596 \\ \mathrm{C} & -5.55222302 & 0.70845179 & -0.64415248 \\ \mathrm{H} & -1.47714312 & 0.92323772 & 1.99241172 \\ \mathrm{H} & -5.35750412 & -1.22153537 & 0.27452209 \\ \mathrm{H} & -3.25856441 & -0.66788992 & 1.45206297 \\ \mathrm{C} & -1.96455596 & 1.64611441 & 1.37210696 \\ \mathrm{C} & -3.24174808 & 3.59621773 & -0.27873172 \\ \mathrm{H} & -5.47238514 & 2.71232153 & -1.40941856 \\ \mathrm{H} & -6.46152127 & 0.46415237 & -1.15247760 \\ \mathrm{C} & -2.06004267 & 3.87136683 & 0.40039341 \\ \mathrm{C} & -1.43834372 & 2.92211479 & 1.20397230 \\ \mathrm{H} & -3.71111966 & 4.33422427 & -0.89512173 \\ \mathrm{C} & -1.22119413 & 5.16628022 & 0.42859260 \\ \mathrm{H} & -1.25234375 & 5.73566936 & -0.47679444 \\ \mathrm{H} & -1.54791079 & 5.79785740 & 1.22813402 \\ \mathrm{C} & 0.16180221 & 4.56814375 & 0.70747254 \\ \mathrm{C} & -0.15892743 & 3.54433954 & 1.80162867 \\ \mathrm{H} & -0.38264494 & 4.01865065 & 2.73430193 \\ \mathrm{H} & 0.63790566 & 2.84950868 & 1.96645312 \\ \mathrm{C} & 1.22819171 & 5.60483716 & 1.10707252 \\ \mathrm{H} & 2.15139056 & 5.10515852 & 1.31425642 \\ \mathrm{H} & 1.37013084 & 6.29803886 & 0.30443645 \\ \mathrm{H} & 0.90398675 & 6.13161408 & 1.98016887 \\ \mathrm{C} & 0.66061209 & 3.83096558 & -0.54925216 \\ \mathrm{H} & -0.06497183 & 3.10330201 & -0.84746971 \\ \mathrm{H} & 0.80606196 & 4.53513994 & -1.34164271 \\ \mathrm{H} & 1.58732183 & 3.34225954 & -0.33182287 \\ \mathrm{H} & -19 & \end{array}$

Figure S11. Cartesian coordinates of the S0 optimized structure of compound $\mathbf{4 j}$. 

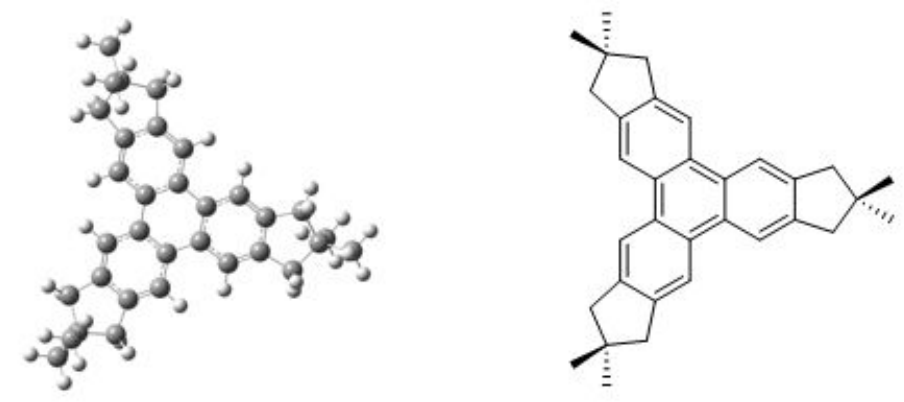

Total Energy $=-1278.827413$ Hartree HOMO: -0.20625 Hartree $=-5.6120625 \mathrm{eV}$ LUMO: -0.03638 Hartree $=-0.9898998 \mathrm{eV}$ Cartesian coordinates:

$\begin{array}{lrrr}\text { Atoms } & \mathbf{X} & \mathbf{Y} & \mathbf{Z} \\ \mathrm{C} & 3.69224383 & 0.70360538 & 0.23837274 \\ \mathrm{C} & 2.50173499 & 1.39501739 & 0.13684773 \\ \mathrm{C} & 1.26259677 & 0.71197611 & 0.03667032 \\ \mathrm{C} & 1.26254814 & -0.71203888 & 0.03678858 \\ \mathrm{C} & 2.50163976 & -1.39513939 & 0.13714022 \\ \mathrm{C} & 3.69219336 & -0.70378505 & 0.23853811 \\ \mathrm{C} & -0.00316793 & 1.44575304 & -0.07745956 \\ \mathrm{C} & -0.00325976 & -1.44574768 & -0.07733971 \\ \mathrm{C} & -1.23037130 & -0.73373298 & -0.20033662 \\ \mathrm{C} & -1.23033069 & 0.73380661 & -0.20034373 \\ \mathrm{C} & -2.43832643 & -1.46883584 & -0.31192265 \\ \mathrm{H} & -3.38508424 & -0.94927679 & -0.40206600 \\ \mathrm{C} & -2.43795777 & -2.84926715 & -0.30174927 \\ \mathrm{C} & -1.22580603 & -3.55289045 & -0.17426720 \\ \mathrm{C} & -0.03484271 & -2.86376748 & -0.06275483 \\ \mathrm{H} & 2.52815666 & 2.47836528 & 0.12702088 \\ \mathrm{H} & 2.52799335 & -2.47849001 & 0.12755978 \\ \mathrm{H} & 0.88472647 & -3.42733293 & 0.04320255 \\ \mathrm{C} & -0.03466201 & 2.86377279 & -0.06292454 \\ \mathrm{C} & -2.43825254 & 1.46897755 & -0.31181964 \\ \mathrm{C} & -1.22559411 & 3.55296415 & -0.17436082 \\ \mathrm{C} & -2.43780067 & 2.84941074 & -0.30168941 \\ \mathrm{H} & -3.38505298 & 0.94946957 & -0.40180634 \\ \mathrm{H} & 0.88494774 & 3.42728533 & 0.04297870 \\ \mathrm{C} & -3.59227533 & -3.81913386 & -0.41912167 \\ \mathrm{H} & -4.48554227 & -3.50318491 & 0.13288447 \\ \mathrm{H} & -3.89020698 & -3.92649130 & -1.47262787 \\ \mathrm{C} & -1.48456825 & -5.04272577 & -0.19541707 \\ \mathrm{H} & -1.26686745 & -5.45020345 & -1.19377679 \\ \mathrm{H} & -0.86766169 & -5.60277594 & 0.51749712 \\ \mathrm{C} & -3.01114593 & -5.16769445 & 0.11671064 \\ \mathrm{C} & -3.64771733 & -6.38805619 & -0.56054637 \\ \mathrm{H} & -3.20061815 & -7.32026547 & -0.19493056 \\ \mathrm{H} & -4.72418139 & -6.43577300 & -0.35673322 \\ \mathrm{H} & -3.51310690 & -6.35514946 & -1.64799921 \\ & & & \\ & & & \end{array}$




$\begin{array}{lrrr}\mathrm{C} & -3.22050391 & -5.24943274 & 1.64179011 \\ \mathrm{H} & -4.28851930 & -5.27200992 & 1.88844319 \\ \mathrm{H} & -2.76219870 & -6.15768755 & 2.05049087 \\ \mathrm{H} & -2.77518379 & -4.38855543 & 2.15256174 \\ \mathrm{C} & 5.10662351 & -1.22368122 & 0.36516062 \\ \mathrm{H} & 5.30818910 & -1.52206984 & 1.40462913 \\ \mathrm{H} & 5.30685245 & -2.10088905 & -0.26163845 \\ \mathrm{C} & 5.10671531 & 1.22342956 & 0.36485584 \\ \mathrm{H} & 5.30831002 & 1.52207361 & 1.40424614 \\ \mathrm{H} & 5.30700666 & 2.10045968 & -0.26217318 \\ \mathrm{C} & 5.99715698 & -0.00020853 & -0.02658902 \\ \mathrm{C} & 6.23174734 & -0.00040288 & -1.55018930 \\ \mathrm{H} & 5.28406115 & -0.00045095 & -2.09991142 \\ \mathrm{H} & 6.79953622 & 0.88560920 & -1.85776418 \\ \mathrm{H} & 6.79950261 & -0.88651150 & -1.85754755 \\ \mathrm{C} & 7.34488163 & -0.00016727 & 0.70602643 \\ \mathrm{H} & 7.20686993 & -0.00005872 & 1.79355257 \\ \mathrm{H} & 7.93718158 & -0.88474993 & 0.44280533 \\ \mathrm{H} & 7.93721899 & 0.88433947 & 0.44263499 \\ \mathrm{C} & -1.48426437 & 5.04281618 & -0.19553338 \\ \mathrm{H} & -0.86725091 & 5.60284441 & 0.51730685 \\ \mathrm{H} & -1.26662940 & 5.45025324 & -1.19392299 \\ \mathrm{C} & -3.59207642 & 3.81933979 & -0.41895369 \\ \mathrm{H} & -3.89014792 & 3.92666263 & -1.47242242 \\ \mathrm{H} & -4.48528588 & 3.50346882 & 0.13319091 \\ \mathrm{C} & -3.01080056 & 5.16789159 & 0.11675101 \\ \mathrm{C} & -3.64736313 & 6.38826516 & -0.56049406 \\ \mathrm{H} & -3.20010628 & 7.32045491 & -0.19502157 \\ \mathrm{H} & -3.51293335 & 6.35526350 & -1.64796618 \\ \mathrm{H} & -4.72378875 & 6.43610771 & -0.35650689 \\ \mathrm{C} & -3.21998859 & 5.24970596 & 1.64184791 \\ \mathrm{H} & -4.28797538 & 5.27245024 & 1.88861034 \\ \mathrm{H} & -2.77474397 & 4.38877318 & 2.15259303 \\ \mathrm{H} & -2.76150213 & 6.15790028 & 2.05047989\end{array}$

Figure S12. Cartesian coordinates of the S0 optimized structure of compound $6 \mathbf{6}$.
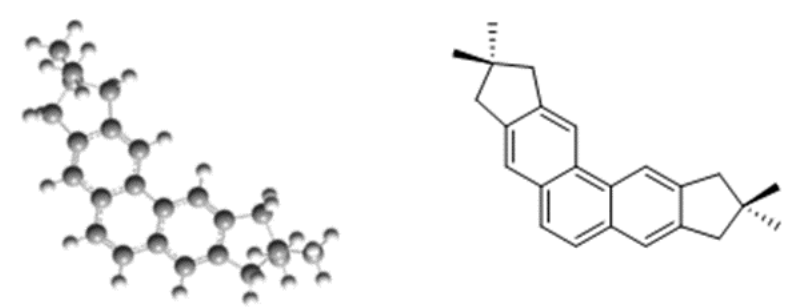

Total Energy $=-929.952254$ Hartree

HOMO: -0.20784 Hartree $=-5.6553264 \mathrm{eV}$

LUMO: -0.03990 Hartree $=-1.085679 \mathrm{eV}$ 
Cartesian coordinates:

$\begin{array}{lrrr}\text { Atoms } & \mathbf{X} & \mathbf{Y} & \mathbf{Z} \\ \mathrm{C} & 2.83672351 & 1.81046029 & -0.22005928 \\ \mathrm{C} & 1.42164862 & 1.78831280 & -0.11336896 \\ \mathrm{C} & 0.72713059 & 0.53950472 & -0.05770423 \\ \mathrm{C} & 1.49238281 & -0.65505058 & -0.11021578 \\ \mathrm{C} & 0.67906813 & 3.01495737 & -0.05372110 \\ \mathrm{C} & -0.72703390 & 0.53953629 & 0.05740127 \\ \mathrm{C} & -1.42149461 & 1.78835401 & 0.11328441 \\ \mathrm{C} & -0.67887134 & 3.01498099 & 0.05377368 \\ \mathrm{C} & -2.83657362 & 1.81055625 & 0.22006003 \\ \mathrm{H} & -3.34266598 & 2.77321615 & 0.25443085 \\ \mathrm{C} & -1.49232093 & -0.65501253 & 0.10973648 \\ \mathrm{H} & 1.22749494 & 3.95259771 & -0.09678356 \\ \mathrm{H} & 3.34286723 & 2.77310267 & -0.25427369 \\ \mathrm{H} & 0.99935778 & -1.62033036 & -0.06105775 \\ \mathrm{H} & -1.22725872 & 3.95263985 & 0.09690066 \\ \mathrm{H} & -0.99929699 & -1.62028516 & 0.06040101 \\ \mathrm{C} & -2.86804687 & -0.60715799 & 0.21657221 \\ \mathrm{C} & -3.54891275 & 0.63170406 & 0.27256581 \\ \mathrm{C} & -3.86330034 & -1.74358009 & 0.29970406 \\ \mathrm{H} & -3.61894811 & -2.58527060 & -0.35917567 \\ \mathrm{H} & -3.89706917 & -2.14065575 & 1.32510217 \\ \mathrm{C} & -5.03781541 & 0.39797367 & 0.39562676 \\ \mathrm{H} & -5.63886154 & 1.09968545 & -0.19468992 \\ \mathrm{H} & -5.35192486 & 0.51230224 & 1.44365463 \\ \mathrm{C} & -5.23097522 & -1.08242902 & -0.06523031 \\ \mathrm{C} & 2.86811337 & -0.60723258 & -0.21705027 \\ \mathrm{H} & 3.54904458 & 0.63158844 & -0.27276673 \\ \mathrm{C} & 3.86337672 & -1.74360543 & -0.30034551 \\ \mathrm{C} & 3.358772739 & -2.13999203 & -1.32599480 \\ \mathrm{H} & 3.61889686 & -2.58587477 & 0.35772920 \\ \mathrm{H} & 5.03800825 & 0.39796449 & -0.39533360 \\ \mathrm{C} & 5.63867511 & 1.09965099 & 0.19542757 \\ \mathrm{H} & 5.35276916 & 0.51252601 & -1.44313805 \\ \mathrm{H} & 5.23094577 & -1.08250808 & 0.06543984 \\ \mathrm{C} & -5.43404312 & -1.12546773 & -1.59273320 \\ \mathrm{C} & -5.50594253 & -2.16026177 & -1.94773327 \\ \mathrm{H} & -6.35716277 & -0.60757247 & -1.87834722 \\ \mathrm{H} & -4.60209320 & -0.64583817 & -2.12007283 \\ \mathrm{H} & -6.41411838 & -1.76275137 & 0.63502899 \\ \mathrm{H} & -7.35810265 & -1.25998913 & 0.39284818 \\ \mathrm{H} & -6.50813566 & -2.80990813 & 0.32329005 \\ \mathrm{H} & -6.29492708 & -1.74632472 & 1.72465495 \\ \mathrm{H} & 5.50402342 & -2.16007720 & 1.94849657 \\ \mathrm{H} & & -1.26038838 & -0.39145960\end{array}$




$\begin{array}{llll}\mathrm{H} & 6.50803655 & -2.81013121 & -0.32242140 \\ \mathrm{H} & 6.29579488 & -1.74636657 & -1.72383595\end{array}$

Figure S13. Cartesian coordinates of the S0 optimized structure of compound $\mathbf{6 b}$.
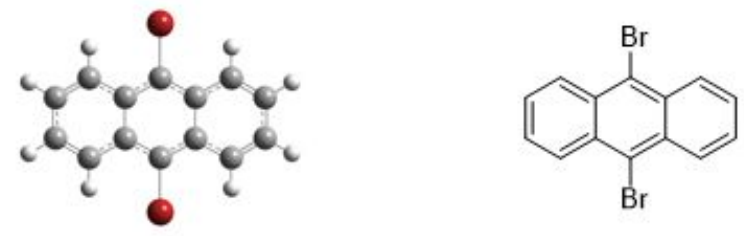

Total Energy $=-5681.679284$ Hartree

HOMO: -0.21339 Hartree $=-5.8063419 \mathrm{eV}$

LUMO: -0.09145 Hartree $=-2.4883545 \mathrm{eV}$

Cartesian coordinates:

$\begin{array}{lccc}\text { Atoms } & \mathbf{X} & \mathbf{Y} & \mathbf{Z} \\ \mathrm{C} & -8.54251986 & -3.33400766 & -0.01291600 \\ \mathrm{C} & -7.17789686 & -3.33400766 & -0.01291600 \\ \mathrm{C} & -6.44500586 & -2.10278666 & -0.01291600 \\ \mathrm{C} & -7.16900286 & -0.87103966 & -0.01843400 \\ \mathrm{C} & -8.60124286 & -0.91253066 & -0.01973400 \\ \mathrm{C} & -9.26502586 & -2.10482366 & -0.01636300 \\ \mathrm{C} & -5.04610486 & -2.07989766 & -0.00802000 \\ \mathrm{C} & -6.46862586 & 0.34012734 & -0.02015500 \\ \mathrm{C} & -5.06968486 & 0.36301134 & -0.01453000 \\ \mathrm{C} & -4.34571586 & -0.86869166 & -0.00711000 \\ \mathrm{C} & -2.91353386 & -0.82706766 & 0.00000000 \\ \mathrm{H} & -2.36766386 & -1.78259466 & 0.00660100 \\ \mathrm{C} & -2.24979686 & 0.36528734 & 0.00104200 \\ \mathrm{C} & -2.97227986 & 1.59440234 & -0.00713000 \\ \mathrm{C} & -4.33692286 & 1.59425734 & -0.01449700 \\ \mathrm{H} & -9.10839186 & -4.27728566 & -0.01051200 \\ \mathrm{H} & -6.60857086 & -4.27574166 & -0.00981000 \\ \mathrm{H} & -9.14705586 & 0.04301534 & -0.02433800 \\ \mathrm{H} & -10.36442386 & -2.14098866 & -0.01779400 \\ \mathrm{H} & -1.15039086 & 0.40119134 & 0.00720200 \\ \mathrm{H} & -2.40619986 & 2.53757934 & -0.00603500 \\ \mathrm{H} & -4.90646586 & 2.53587434 & -0.02076900 \\ \mathrm{Br} & -7.43691732 & 1.98647385 & -0.02755937 \\ \mathrm{Br} & -4.07856975 & -3.72668838 & -0.00052715\end{array}$

Figure S14. Cartesian coordinates of the S0 optimized structure of 9,10-Dibromoanthracene. 

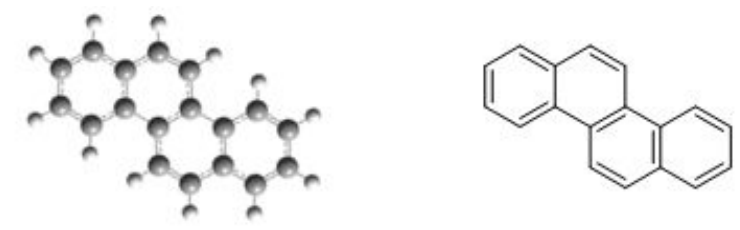

Total Energy $=-693.020562$ Hartree

HOMO: -0.21362 Hartree $=-5.8126002 \mathrm{eV}$

LUMO: -0.05913 Hartree $=-1.6089273 \mathrm{eV}$

Cartesian coordinates:

$\begin{array}{lrrr}\text { Atoms } & \mathbf{X} & \mathbf{Y} & \mathbf{Z} \\ \mathrm{C} & 1.07479299 & -4.58656896 & 0.74753856 \\ \mathrm{C} & 0.49767530 & -3.32945482 & 0.55985360 \\ \mathrm{C} & -0.85889769 & -3.13032058 & 0.83915938 \\ \mathrm{C} & -1.60971647 & -4.15211384 & 1.31898722 \\ \mathrm{C} & 2.43049255 & -4.79559916 & 0.44741743 \\ \mathrm{C} & 1.28068645 & -2.27342925 & 0.09845587 \\ \mathrm{C} & 2.65482921 & -2.49181161 & -0.16405664 \\ \mathrm{C} & 3.20429989 & -3.77081196 & 0.00543330 \\ \mathrm{C} & 3.46799304 & -1.42499943 & -0.59273694 \\ \mathrm{H} & 4.51149543 & -1.56397798 & -0.78455972 \\ \mathrm{C} & 2.88508589 & -0.22405392 & -0.75379539 \\ \mathrm{C} & 1.52617525 & -0.01489796 & -0.51727682 \\ \mathrm{C} & 0.71098627 & -0.99956665 & -0.10032011 \\ \mathrm{H} & 2.85893136 & -5.76821821 & 0.57027057 \\ \mathrm{H} & -1.30293749 & -2.17063790 & 0.67573072 \\ \mathrm{H} & -2.64580192 & -3.99350697 & 1.53499673 \\ \mathrm{H} & 4.23828336 & -3.93956732 & -0.21424217 \\ \mathrm{H} & -0.32985003 & -0.82195925 & 0.07596852 \\ \mathrm{C} & -1.03537350 & -5.41535015 & 1.53626380 \\ \mathrm{C} & 0.29278170 & -5.63312426 & 1.24161869 \\ \mathrm{C} & -1.83017409 & -6.45808543 & 2.06003780 \\ \mathrm{C} & -1.22699157 & -7.69524050 & 2.25552410 \\ \mathrm{C} & 0.87086592 & -6.90340682 & 1.44486643 \\ \mathrm{C} & 0.05315290 & -7.89649098 & 1.97102269 \\ \mathrm{H} & 1.89672941 & -7.09102466 & 1.20662178 \\ \mathrm{H} & -2.86096692 & -6.30141903 & 2.29669491 \\ \mathrm{H} & 1.29815981 & 1.00994023 & -0.72371241 \\ \mathrm{H} & 3.32203365 & 0.70090760 & -1.06751953 \\ \mathrm{H} & 0.26354408 & -8.90959405 & 2.24352540 \\ \mathrm{H} & -1.65090536 & -8.60838017 & 2.61797830\end{array}$

Figure S15. Cartesian coordinates of the S0 optimized structure of Chrysene.
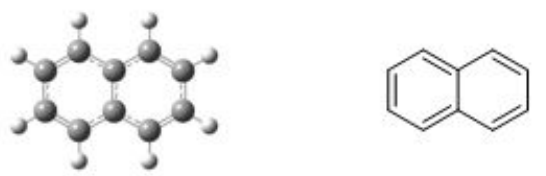

Total Energy $=-385.803409$ Hartree 
HOMO: -0.22364 Hartree $=-6.0852444 \mathrm{eV}$

LUMO: -0.04964 Hartree $=-1.3507044 \mathrm{eV}$

Cartesian coordinates:

$\begin{array}{lrrr}\text { Atoms } & \mathbf{X} & \mathbf{Y} & \mathbf{Z} \\ \mathrm{C} & 2.43637422 & -0.70913873 & -0.00000194 \\ \mathrm{C} & 1.24592694 & -1.40380499 & 0.00000207 \\ \mathrm{C} & 0.00001401 & -0.71726892 & 0.00000133 \\ \mathrm{C} & -0.00001637 & 0.71726376 & 0.00000143 \\ \mathrm{C} & 1.24590786 & 1.40380724 & 0.00000312 \\ \mathrm{C} & 2.43635872 & 0.70915816 & -0.00000204 \\ \mathrm{H} & -1.24432101 & -2.49097353 & 0.00000797 \\ \mathrm{H} & 3.38034285 & -1.24643868 & -0.00000462 \\ \mathrm{H} & 1.24435156 & -2.49096230 & -0.00000047 \\ \mathrm{C} & -1.24591303 & -1.40381465 & 0.00000039 \\ \mathrm{C} & -1.24592877 & 1.40381238 & -0.00000005 \\ \mathrm{H} & 1.24432559 & 2.49096677 & -0.00000098 \\ \mathrm{H} & 3.38032463 & 1.24646337 & -0.00000550 \\ \mathrm{C} & -2.43636950 & 0.70914128 & -0.00000100 \\ \mathrm{C} & -2.43635577 & -0.70915414 & -0.00000249 \\ \mathrm{H} & -1.24433741 & 2.49096754 & 0.00000584 \\ \mathrm{H} & -3.38035108 & 1.24642173 & -0.00000506 \\ \mathrm{H} & -3.38032507 & -1.24645329 & -0.00000201\end{array}$

Figure S16. Cartesian coordinates of the S0 optimized structure of Napthalene.
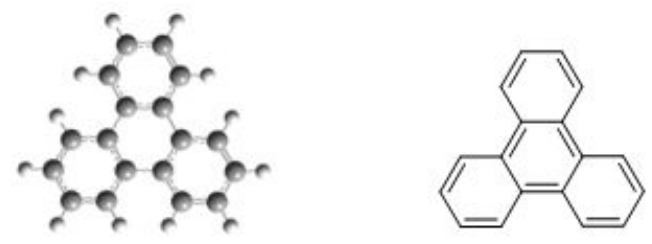

Total Energy $=-693.019930$ Hartree

HOMO: -0.22586 Hartree $=-6.1456506 \mathrm{eV}$

LUMO: -0.04826 Hartree $=-1.3131546 \mathrm{eV}$

Cartesian coordinates:

$\begin{array}{lrrr}\text { Atoms } & \mathbf{X} & \mathbf{Y} & \mathbf{Z} \\ \mathrm{C} & -1.41180182 & -3.74185759 & 0.02618480 \\ \mathrm{C} & 0.01346018 & -3.74185759 & 0.02618480 \\ \mathrm{C} & 0.69603818 & -2.50960659 & 0.02618480 \\ \mathrm{C} & -0.04038882 & -1.28482559 & 0.02636080 \\ \mathrm{C} & -1.44883682 & -1.30983759 & 0.02624780 \\ \mathrm{C} & -2.11721682 & -2.56866259 & 0.02618780 \\ \mathrm{C} & 2.12493018 & -2.48423259 & 0.02623280 \\ \mathrm{C} & 0.65208818 & -0.03468359 & 0.02672880 \\ \mathrm{C} & 2.08098518 & -0.00929859 & 0.02712280 \\ \mathrm{C} & 2.81740618 & -1.23407759 & 0.02683280 \\ \mathrm{C} & 2.76353518 & 1.22295741 & 0.02786380 \\ \mathrm{C} & 2.00753618 & 2.43119641 & 0.02801680 \\ \mathrm{C} & 0.63880918 & 2.40688741 & 0.02747580 \\ \mathrm{C} & -0.07380482 & 1.17256341 & 0.02693980\end{array}$




$\begin{array}{lrrr}\mathrm{H} & -1.93386882 & -4.71093859 & 0.02617880 \\ \mathrm{H} & -3.21795282 & -2.57531659 & 0.02618980 \\ \mathrm{H} & 2.55214418 & 3.38779241 & 0.02852880 \\ \mathrm{H} & 0.06057218 & 3.34353541 & 0.02749480 \\ \mathrm{C} & 2.85081018 & -3.69147059 & 0.02580680 \\ \mathrm{C} & 4.27517618 & -3.64088459 & 0.02583980 \\ \mathrm{C} & 4.93849118 & -2.44337259 & 0.02668380 \\ \mathrm{C} & 4.22584318 & -1.20907459 & 0.02724080 \\ \mathrm{H} & 4.83130818 & -4.59081559 & 0.02534280 \\ \mathrm{H} & 6.03877118 & -2.41094259 & 0.02693380 \\ \mathrm{H} & 3.73723503 & 1.22296083 & 0.02840556 \\ \mathrm{H} & 4.68245695 & -0.34907745 & 0.02792944 \\ \mathrm{H} & 2.36396721 & -4.53472357 & 0.02570774 \\ \mathrm{H} & 0.52992618 & -4.56729962 & 0.02605978 \\ \mathrm{H} & -1.93569639 & -0.46659418 & 0.02629973 \\ \mathrm{H} & -1.04689138 & 1.13800647 & 0.02672802\end{array}$

Figure S17. Cartesian coordinates of the S0 optimized structure of Triphenylene.
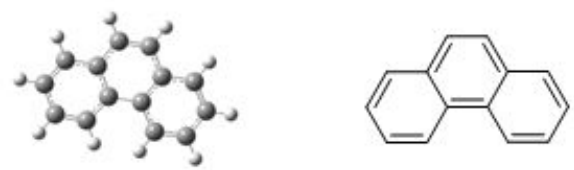

Total Energy $=-539.413073$ Hartree

HOMO: -0.22158 Hartree $=-6.0291918 \mathrm{eV}$

LUMO: -0.04995 Hartree $=-1.3591395 \mathrm{eV}$

Cartesian coordinates:

$\begin{array}{lccc}\text { Atoms } & \mathbf{X} & \mathbf{Y} & \mathbf{Z} \\ \mathrm{C} & -6.28533023 & -5.58954269 & -0.00346100 \\ \mathrm{C} & -4.90543923 & -5.58954269 & -0.00346100 \\ \mathrm{C} & -4.18610323 & -4.37289869 & -0.00346100 \\ \mathrm{C} & -4.88369223 & -3.14100269 & -0.00139100 \\ \mathrm{C} & -6.29767723 & -3.17086669 & -0.00098200 \\ \mathrm{C} & -6.98413923 & -4.36943569 & -0.00246900 \\ \mathrm{C} & -2.75105123 & -4.38138169 & -0.00514100 \\ \mathrm{C} & -4.13426023 & -1.90431569 & 0.00008000 \\ \mathrm{C} & -2.71941323 & -1.95259069 & -0.00173700 \\ \mathrm{C} & -2.04772323 & -3.22076669 & -0.00482900 \\ \mathrm{C} & -1.97368423 & -0.75187969 & 0.00000000 \\ \mathrm{H} & -0.87464923 & -0.80853569 & -0.00141800 \\ \mathrm{C} & -2.61230223 & 0.47130331 & 0.00368100 \\ \mathrm{C} & -4.01731023 & 0.52610931 & 0.00560300 \\ \mathrm{C} & -4.76217523 & -0.63701769 & 0.00368600 \\ \mathrm{H} & -2.24146523 & -5.35715069 & -0.00696600 \\ \mathrm{H} & -6.84455123 & -6.53645769 & -0.00431500 \\ \mathrm{H} & -4.34642023 & -6.53748369 & -0.00416700 \\ \mathrm{H} & -6.86705923 & -2.22989269 & 0.00032200 \\ \mathrm{H} & -8.08423423 & -4.37635869 & -0.00274600 \\ \mathrm{H} & -0.94690623 & -3.22124669 & -0.00645800\end{array}$




$\begin{array}{rrrr}\mathrm{H} & -2.03169423 & 1.40526631 & 0.00514800 \\ \mathrm{H} & -4.52003523 & 1.50463331 & 0.00858400 \\ \mathrm{H} & -5.85983423 & -0.56791269 & 0.00532300\end{array}$

Figure S18. Cartesian coordinates of the S0 optimized structure of Phenanthrene.
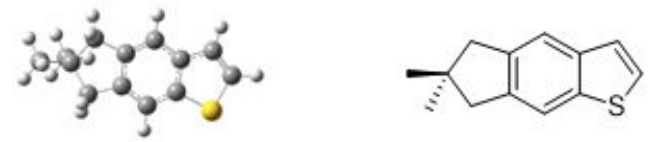

Total Energy $=-901.862183$ Hartree

HOMO: -0.21816 Hartree $=-5.9361336 \mathrm{eV}$

LUMO: -0.03276 Hartree $=-0.8913996 \mathrm{eV}$

Cartesian coordinates:

$\begin{array}{lrrr}\mathrm{C} & -0.75869728 & 0.20420157 & 1.21052425 \\ \mathrm{C} & 0.63443050 & 0.10205876 & 1.07344141 \\ \mathrm{C} & 1.45382629 & 1.23618997 & 0.87174035 \\ \mathrm{C} & 0.93886186 & 2.53128111 & 0.73789183 \\ \mathrm{C} & -0.45063450 & 2.65741075 & 0.84256573 \\ \mathrm{C} & -1.31104482 & 1.49090776 & 1.12962925 \\ \mathrm{H} & -1.37044116 & -0.65883934 & 1.37124082 \\ \mathrm{H} & 1.57175524 & 3.37616288 & 0.56318989 \\ \mathrm{C} & 1.47881879 & -1.20589807 & 1.06657228 \\ \mathrm{H} & 1.67212705 & -1.60297206 & 2.04118203 \\ \mathrm{H} & 0.99266800 & -1.96469843 & 0.48970190 \\ \mathrm{C} & 2.91583621 & 0.79305034 & 0.82530068 \\ \mathrm{H} & 3.32222088 & 0.87500147 & 1.81172630 \\ \mathrm{H} & 3.54274847 & 1.34596615 & 0.15734475 \\ \mathrm{C} & 2.76332462 & -0.68113572 & 0.42471305 \\ \mathrm{C} & 3.87287637 & -1.59686550 & -0.12477206 \\ \mathrm{H} & 4.63488145 & -0.99984492 & -0.58063903 \\ \mathrm{H} & 4.29657275 & -2.16452303 & 0.67719197 \\ \mathrm{H} & 3.45809722 & -2.26248235 & -0.85265425 \\ \mathrm{C} & 3.59264059 & -0.74066796 & 1.72097341 \\ \mathrm{H} & 4.55921732 & -0.31647255 & 1.54579444 \\ \mathrm{H} & 3.09410304 & -0.18715596 & 2.48907808 \\ \mathrm{H} & 3.70081446 & -1.75973868 & 2.02869615 \\ \mathrm{C} & -1.28793123 & 3.95522543 & 0.66780991 \\ \mathrm{H} & -1.00713494 & 4.96617854 & 0.45798318 \\ \mathrm{C} & -2.42795210 & 3.40247934 & 0.84995811 \\ \mathrm{~S} & -3.07648391 & 1.90222355 & 1.34579351 \\ \mathrm{H} & -2.89668795 & 3.57763978 & -0.41181274\end{array}$

Figure S19. Cartesian coordinates of the S0 optimized structure of $4 \mathbf{e}$. 

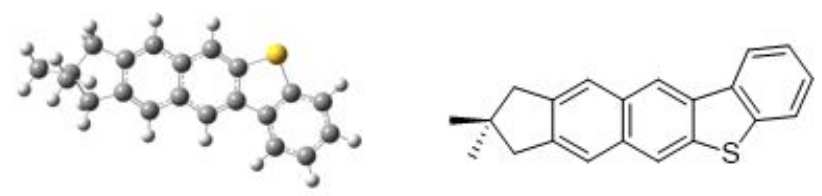

Total Energy $=-1209.085678$ Hartree

HOMO: -0.20334 Hartree $=-5.5328814 \mathrm{eV}$

LUMO: -0.06016 Hartree $=-1.6369536 \mathrm{eV}$

Cartesian coordinates:

$\begin{array}{lrrr}\mathrm{C} & -3.09409780 & 0.55581613 & 0.82944750 \\ \mathrm{C} & -1.76566624 & 0.67356374 & 1.23053027 \\ \mathrm{C} & -0.94832803 & 1.60244365 & 0.56010033 \\ \mathrm{C} & -1.49211230 & 2.40727109 & -0.49831561 \\ \mathrm{C} & -2.85054383 & 2.28607696 & -0.85127309 \\ \mathrm{C} & -3.63636930 & 1.37078566 & -0.15127765 \\ \mathrm{H} & 0.82373506 & 1.15019987 & 1.72521477 \\ \mathrm{H} & -1.37482401 & 0.06985144 & 2.02277238 \\ \mathrm{C} & 0.40746983 & 1.73423815 & 0.93171386 \\ \mathrm{C} & -0.67317335 & 3.32592717 & -1.18843606 \\ \mathrm{H} & -3.27072438 & 2.88379311 & -1.63246980 \\ \mathrm{C} & 0.65951426 & 3.42038323 & -0.80321491 \\ \mathrm{C} & 1.18414159 & 2.64830823 & 0.22848887 \\ \mathrm{H} & -1.06410499 & 3.92901592 & -1.98150490 \\ \mathrm{C} & 1.78057392 & 4.32023925 & -1.36319918 \\ \mathrm{H} & 2.29407801 & 3.81650593 & -2.15508402 \\ \mathrm{H} & 1.44684719 & 5.26654489 & -1.73456300 \\ \mathrm{C} & 2.67666365 & 3.00385550 & 0.40012406 \\ \mathrm{H} & 3.04057234 & 2.92373743 & 1.40299079 \\ \mathrm{H} & 3.27735257 & 2.37299779 & -0.22108544 \\ \mathrm{C} & 2.65559669 & 4.44752284 & -0.11177856 \\ \mathrm{C} & 1.94276445 & 5.34236442 & 0.91847464 \\ \mathrm{H} & 2.51183728 & 5.36531137 & 1.82435097 \\ \mathrm{H} & 1.85297820 & 6.33415406 & 0.52794954 \\ \mathrm{H} & 0.96902094 & 4.94855179 & 1.11947891 \\ \mathrm{C} & 4.05068771 & 5.04270919 & -0.37635047 \\ \mathrm{H} & 4.60636558 & 5.06955233 & 0.53749252 \\ \mathrm{H} & 4.56745866 & 4.43685037 & -1.09006617 \\ \mathrm{H} & 3.94720996 & 6.03595518 & -0.75996284 \\ \mathrm{C} & -5.15679412 & 1.13501023 & -0.31468326 \\ \mathrm{C} & -5.62092266 & 0.19084532 & 0.52494951 \\ \mathrm{~S} & -4.30960977 & -0.58204634 & 1.44081035 \\ \mathrm{C} & -6.09672643 & 1.86604121 & -1.27894874 \\ \mathrm{H} & -5.71894010 & 2.58955700 & -1.97107322 \\ \mathrm{C} & -7.11673001 & -0.12376038 & 0.62005136 \\ \mathrm{H} & -7.48197867 & -0.84681767 & 1.32262812 \\ \mathrm{C} & -7.97265925 & 0.53247835 & -0.21321711 \\ \mathrm{H} & -9.02140311 & 0.32825745 & -0.16917255 \\ \mathrm{C} & -7.42782114 & 1.57629404 & -1.23149267 \\ \mathrm{H} & -8.10388198 & 2.07256096 & -1.89680133 \\ \mathrm{H} & -\mathrm{s} & & \end{array}$

Figure S20. Cartesian coordinates of the S0 optimized structure of $\mathbf{4 f a}$. 

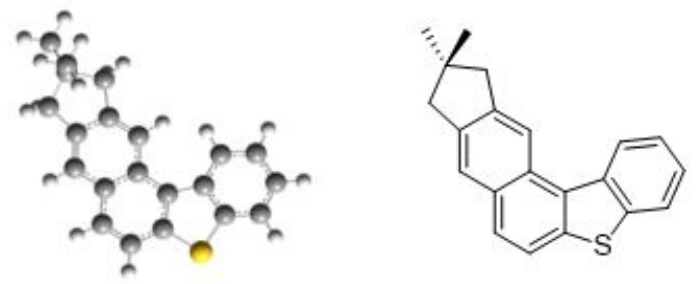

Total Energy $=-1209.082809$ Hartree HOMO: -0.21102 Hartree $=-5.7418542 \mathrm{eV}$

LUMO: -0.05739 Hartree $=-1.5615819 \mathrm{eV}$

Cartesian coordinates:

$\begin{array}{lccc}\mathrm{C} & -1.55871055 & -2.66864738 & -0.55312268 \\ \mathrm{C} & -0.15281147 & -2.67289701 & -0.41041722 \\ \mathrm{C} & 0.53489146 & -1.46149428 & -0.21248671 \\ \mathrm{C} & -0.18349220 & -0.32087998 & -0.16750546 \\ \mathrm{C} & -1.57301143 & -0.32216572 & -0.24587770 \\ \mathrm{C} & -2.26926603 & -1.45699900 & -0.46435476 \\ \mathrm{H} & -2.08251449 & -3.58554930 & -0.72580821 \\ \mathrm{H} & 0.38780876 & -3.59520070 & -0.45497796 \\ \mathrm{H} & 1.59939628 & -1.44507351 & -0.10543599 \\ \mathrm{H} & -3.33455069 & -1.43701755 & -0.56268753 \\ \mathrm{C} & -1.16705725 & 2.03890562 & 0.19240706 \\ \mathrm{C} & -1.45433754 & 3.31803322 & 0.51673624 \\ \mathrm{C} & -2.80587298 & 3.70934563 & 0.56210214 \\ \mathrm{C} & -3.81874866 & 2.76849544 & 0.27379981 \\ \mathrm{C} & -3.46903236 & 1.42192820 & -0.02529604 \\ \mathrm{C} & -2.16206577 & 1.09014540 & -0.03060957 \\ \mathrm{H} & -0.67360861 & 4.01821524 & 0.72912147 \\ \mathrm{H} & -3.06474495 & 4.71708171 & 0.81180859 \\ \mathrm{~S} & 0.44955441 & 1.33088897 & -0.00794740 \\ \mathrm{C} & -4.56525030 & 0.36974533 & -0.32732284 \\ \mathrm{H} & -4.31766848 & -0.64688220 & -0.55108994 \\ \mathrm{C} & -5.30786775 & 3.19225487 & 0.28272371 \\ \mathrm{H} & -5.60302638 & 4.19716541 & 0.50166704 \\ \mathrm{C} & -5.84342511 & 0.79101251 & -0.29285294 \\ \mathrm{C} & -6.21302550 & 2.23815665 & -0.00052538 \\ \mathrm{C} & -7.16390843 & 0.00141610 & -0.51139010 \\ \mathrm{H} & -7.08220150 & -0.81467492 & -1.19857233 \\ \mathrm{H} & -7.50322322 & -0.38301466 & 0.42774698 \\ \mathrm{C} & -7.73265917 & 2.29132512 & -0.11884995 \\ \mathrm{H} & -7.70612785 & 2.88261609 & -1.01023711 \\ \mathrm{H} & -8.36268678 & 3.08349819 & 0.22817606 \\ \mathrm{C} & -8.10420847 & 1.11867818 & -1.03079202 \\ \mathrm{C} & -9.59546419 & 0.73559364 & -0.99912801 \\ \mathrm{H} & -9.87811819 & 0.48484070 & 0.00193626 \\ \mathrm{H} & -10.18339676 & 1.56235887 & -1.33926703 \\ \mathrm{H} & -9.76100985 & -0.10658778 & -1.63805296 \\ \mathrm{C} & -7.73336373 & 1.47643492 & -2.48202746 \\ \mathrm{H} & -7.91305227 & 0.63230039 & -3.11452168 \\ \mathrm{H} & -8.33084119 & 2.30133729 & -2.80983344\end{array}$


Figure S21. Cartesian coordinates of the S0 optimized structure of $\mathbf{4 f b}$.
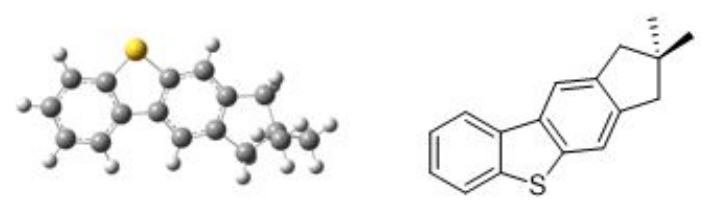

Total Energy $=-1055.478997$ Hartree

HOMO: -0.21574 Hartree $=-5.8702854 \mathrm{eV}$

LUMO: -0.04454 Hartree $=-1.2119334 \mathrm{eV}$

Cartesian coordinates:

$\mathrm{C}$

$\mathrm{C}$

$\mathrm{C}$

$\mathrm{C}$

$\mathrm{C}$

$\mathrm{C}$

C

C

$\mathrm{H}$

$\mathrm{H}$

$\mathrm{H}$

$\mathrm{H}$

S

C

$\mathrm{H}$

C

$\mathrm{H}$

C

C

C

$\mathrm{H}$

$\mathrm{H}$

C

$\mathrm{H}$

$\mathrm{H}$

C

C

$\mathrm{H}$

$\mathrm{H}$

$\mathrm{H}$

C

$\mathrm{H}$

$\mathrm{H}$

$\mathrm{H}$

$\begin{array}{rrc}-0.69012153 & -1.15609293 & 0.11881086 \\ -2.02515631 & -1.24799055 & -0.00446700 \\ -2.88072594 & -0.00562518 & -0.27469705 \\ -2.26271631 & 1.20798651 & -0.32918734 \\ -0.72656287 & 1.31905248 & -0.11513139 \\ 0.02145943 & 0.20037255 & 0.09780541 \\ -1.51678846 & -3.56439797 & 0.42968808 \\ -2.53283558 & -2.69751054 & 0.18246523 \\ -3.93858316 & -0.08834629 & -0.41252061 \\ -2.83849269 & 2.08994073 & -0.51770343 \\ -0.25414002 & 2.27885353 & -0.13746074 \\ 1.07960304 & 0.26715283 & 0.24193201 \\ 0.05730857 & -2.75654168 & 0.29740601 \\ -3.99796121 & -3.17192559 & 0.13379152 \\ -4.79965420 & -2.50568936 & -0.10769892 \\ -1.77719080 & -5.04341378 & 0.77180602 \\ -0.98041090 & -5.71939807 & 1.00221225 \\ -4.21542049 & -4.47810558 & 0.42086195 \\ -3.07170784 & -5.44120110 & 0.75696379 \\ -5.55251122 & -5.23739144 & 0.42957458 \\ -6.11870609 & -5.02624981 & 1.31260492 \\ -6.15510518 & -4.99722312 & -0.42136590 \\ -3.63278543 & -6.85240577 & 1.00004295 \\ -3.66038328 & -7.09611740 & 2.04155292 \\ -3.05745150 & -7.60021510 & 0.49539250 \\ -5.07088639 & -6.70556824 & 0.45369964 \\ -6.06557222 & -7.88038376 & 0.40888213 \\ -6.64763821 & -7.82092226 & -0.48697697 \\ -5.52673664 & -8.80469134 & 0.42343214 \\ -6.71345471 & -7.83180559 & 1.25905179 \\ -5.23938064 & -6.55166482 & 1.97669781 \\ -4.94038669 & -7.45722969 & 2.46193474 \\ -4.62983253 & -5.74336040 & 2.32310085 \\ -6.26499339 & -6.34747147 & 2.20324487\end{array}$

Figure S22. Cartesian coordinates of the S0 optimized structure of $\mathbf{4 g}$. 

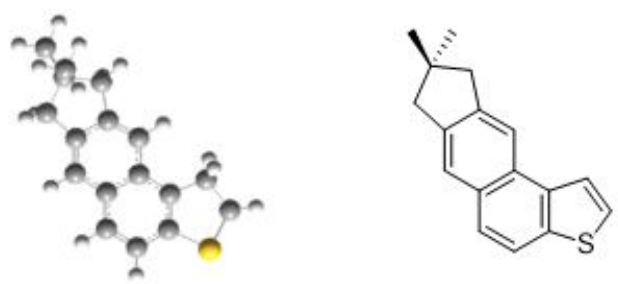

Total Energy $=-1056.658629$ Hartree

HOMO: -0.19824 Hartree $=-5.3941104 \mathrm{eV}$

LUMO: -0.04669 Hartree $=-1.2704349 \mathrm{eV}$

Cartesian coordinates :

$\begin{array}{lrrr}\mathrm{C} & -2.73163228 & -0.48229113 & -1.08453851 \\ \mathrm{C} & -1.43747484 & -0.44641251 & -0.57290841 \\ \mathrm{C} & -0.70447696 & 0.75036620 & -0.72686988 \\ \mathrm{C} & -1.29361562 & 1.87083147 & -1.38734870 \\ \mathrm{C} & -2.60776000 & 1.80713546 & -1.89538856 \\ \mathrm{C} & -3.30088297 & 0.61337010 & -1.72761845 \\ \mathrm{H} & 1.05213615 & -0.00955419 & 0.27109300 \\ \mathrm{H} & -1.01310661 & -1.29614187 & -0.08018980 \\ \mathrm{C} & 0.61716835 & 0.83391784 & -0.22313160 \\ \mathrm{C} & -0.53261318 & 3.03038162 & -1.51561783 \\ \mathrm{H} & -3.05381746 & 2.64434986 & -2.39036814 \\ \mathrm{C} & 0.76505697 & 3.10089651 & -1.01731157 \\ \mathrm{C} & 1.36261030 & 2.02134605 & -0.36919580 \\ \mathrm{H} & 2.36155298 & 2.09203755 & 0.00765461 \\ \mathrm{~S} & 1.47358249 & 4.69742320 & -1.34158975 \\ \mathrm{C} & -0.95893854 & 4.35917509 & -2.18472512 \\ \mathrm{H} & -1.38871762 & 5.00344159 & -1.44640906 \\ \mathrm{H} & -1.66758803 & 4.22672740 & -2.97540427 \\ \mathrm{C} & 0.37385269 & 4.93242000 & -2.70845965 \\ \mathrm{H} & 0.71686591 & 4.34854154 & -3.53690873 \\ \mathrm{C} & -4.73312271 & 0.23945057 & -2.16331820 \\ \mathrm{H} & -4.71911005 & -0.18368904 & -3.14599644 \\ \mathrm{H} & -5.42458688 & 1.05601414 & -2.16442579 \\ \mathrm{C} & -3.76212028 & -1.63089164 & -1.06590441 \\ \mathrm{H} & -3.65407345 & -2.23506722 & -1.94237332 \\ \mathrm{H} & -3.69706747 & -2.27209974 & -0.21178480 \\ \mathrm{C} & -5.05748671 & -0.81272858 & -1.09759146 \\ \mathrm{C} & -5.25353642 & -0.11672531 & 0.26209378 \\ \mathrm{H} & -6.11182130 & 0.52055851 & 0.21608942 \\ \mathrm{H} & -5.39779121 & -0.85491161 & 1.02312699 \\ \mathrm{H} & -4.38721312 & 0.46776384 & 0.49178131 \\ \mathrm{C} & -6.32012374 & -1.64270690 & -1.39505358 \\ \mathrm{H} & -6.45780030 & -2.37146609 & -0.62378500 \\ \mathrm{H} & -7.17181825 & -0.99599491 & -1.43083152 \\ \mathrm{H} & -6.20803943 & -2.13733297 & -2.33722244 \\ \mathrm{H} & 0.29258950 & 5.95251118 & -3.02104610\end{array}$

Figure S23. Cartesian coordinates of the S0 optimized structure of $\mathbf{4 h}$. 

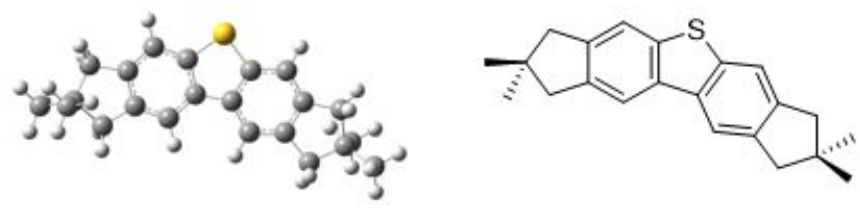

Total Energy $=-1250.748024$ Hartree

HOMO: -0.20996 Hartree $=-5.7130116 \mathrm{eV}$

LUMO: -0.04051 Hartree $=-1.1022771 \mathrm{eV}$

Cartesian coordinates:

$\begin{array}{lccc}\mathrm{C} & -10.98721910 & -5.86302277 & 1.00897464 \\ \mathrm{C} & -9.74660993 & -6.50251331 & 0.85317898 \\ \mathrm{C} & -8.53693986 & -5.80382249 & 0.91181108 \\ \mathrm{C} & -8.63807822 & -4.41938707 & 1.12396636 \\ \mathrm{C} & -9.87895400 & -3.78553020 & 1.33220707 \\ \mathrm{C} & -11.09170280 & -4.48908653 & 1.24813936 \\ \mathrm{H} & -7.59238699 & -6.29309054 & 0.79633792 \\ \mathrm{H} & -12.03817222 & -4.00412288 & 1.36576692 \\ \mathrm{C} & -9.70574062 & -2.28974485 & 1.67515361 \\ \mathrm{C} & -8.34939099 & -1.91142879 & 1.69156177 \\ \mathrm{C} & -7.94143514 & -0.62391043 & 2.07689711 \\ \mathrm{C} & -8.96691040 & 0.28245557 & 2.36663976 \\ \mathrm{C} & -10.31286095 & -0.09285809 & 2.34121211 \\ \mathrm{C} & -10.72910783 & -1.37556433 & 1.97148646 \\ \mathrm{H} & -6.90880657 & -0.34964376 & 2.13475757 \\ \mathrm{H} & -11.76326676 & -1.64670594 & 1.92784716 \\ \mathrm{~S} & -7.30213765 & -3.24880117 & 1.16993185 \\ \mathrm{C} & -8.86298484 & 1.78129980 & 2.73082626 \\ \mathrm{H} & -7.99299603 & 2.05431555 & 3.29072323 \\ \mathrm{H} & -8.86309245 & 2.32585535 & 1.80975941 \\ \mathrm{C} & -11.14019381 & 1.09940771 & 2.83003951 \\ \mathrm{H} & -11.10601949 & 0.53761684 & 3.74005593 \\ \mathrm{H} & -12.20871909 & 1.13680887 & 2.87203884 \\ \mathrm{C} & -10.17899015 & 2.06513625 & 3.51295414 \\ \mathrm{C} & -9.99947273 & -8.00562455 & 0.61611369 \\ \mathrm{H} & -9.27486558 & -8.48394958 & -0.00919568 \\ \mathrm{H} & -10.02520809 & -8.52596593 & 1.55076630 \\ \mathrm{C} & -12.10199957 & -6.92076839 & 0.87018585 \\ \mathrm{H} & -12.33117081 & -7.33935618 & 1.82788039 \\ \mathrm{H} & -13.01160672 & -6.55322632 & 0.44302565 \\ \mathrm{C} & -11.38726513 & -7.93276848 & -0.03240901 \\ \mathrm{C} & -11.25590082 & -7.34634743 & -1.45031607 \\ \mathrm{H} & -10.68584460 & -8.01461760 & -2.06127877 \\ \mathrm{H} & -12.22964465 & -7.21789468 & -1.87476829 \\ \mathrm{H} & -10.76095995 & -6.39905684 & -1.40004815 \\ \mathrm{C} & -12.10249264 & -9.29219313 & -0.14210759 \\ \mathrm{H} & -13.07114248 & -9.15171039 & -0.57439180 \\ \mathrm{H} & -11.52750810 & -9.94884573 & -0.76109326 \\ \mathrm{H} & -12.20577708 & -9.72055816 & 0.83295198 \\ \mathrm{C} & -10.55171562 & 3.55610624 & 3.41430717 \\ \mathrm{H} & -11.48861116 & 3.72268264 & 3.90357153 \\ \mathrm{H} & -9.79329419 & 4.14533003 & 3.88600586\end{array}$




$\begin{array}{cccc}\mathrm{H} & -10.63223505 & 3.83624067 & 2.38476683 \\ \mathrm{C} & -10.09809408 & 1.69241226 & 5.00499295 \\ \mathrm{H} & -9.36430052 & 2.30460699 & 5.48623896 \\ \mathrm{H} & -11.05141253 & 1.85066710 & 5.46433801 \\ \mathrm{H} & -9.82238989 & 0.66306760 & 5.10108065\end{array}$

Figure S24. Cartesian coordinates of the S0 optimized structure of $4 \mathbf{i}$

\section{References}

(1) A. E. Morrison, J. J. Hrudka and G. B. Dudley, Org. Lett., 2016, 18, 4104.

(2) T. T. Hoang, G. B. Dudley, Org. Lett., 2013, 15, 4026.

(3) P. G. Theobald, W. H. Okamura, J. Org. Chem., 1990, 55, 741. 